\title{
Contemporaneous observations of the radio galaxy NGC 1275 from radio to very high energy $\gamma$-rays
}

J. Aleksić ${ }^{1}$, S. Ansoldi ${ }^{2}$, L. A. Antonelli ${ }^{3}$, P. Antoranz ${ }^{4}$, A. Babic ${ }^{5}$, P. Bangale ${ }^{6}$, U. Barres de Almeida ${ }^{6}$, J. A. Barrio ${ }^{7}$, J. Becerra González ${ }^{8}$, W. Bednarek ${ }^{9}$, K. Berger ${ }^{8}$, E. Bernardini ${ }^{10}$, A. Biland ${ }^{11}$, O. Blanch ${ }^{1}$, R. K. Bock ${ }^{6}$, S. Bonnefoy ${ }^{7}$, G. Bonnoli ${ }^{3}$, F. Borracci ${ }^{6}$, T. Bretz ${ }^{12,25}$, E. Carmona ${ }^{13}$, A. Carosi ${ }^{3}$, D. Carreto Fidalgo ${ }^{12}$, P. Colin ${ }^{6, \star}$, E. Colombo $^{8}$, J. L. Contreras ${ }^{7}$, J. Cortina ${ }^{1}$, S. Covino ${ }^{3}$, P. Da Vela ${ }^{4}$, F. Dazzi ${ }^{2}$, A. De Angelis ${ }^{2}$, G. De Caneva ${ }^{10}$, B. De Lotto ${ }^{2}$, C. Delgado Mendez ${ }^{13}$, M. Doert ${ }^{14}$, A. Domínguez ${ }^{15,26}$, D. Dominis Prester ${ }^{5}$, D. Dorner ${ }^{12}$, M. Doro ${ }^{16}, S$. Einecke ${ }^{14}$, D. Eisenacher ${ }^{12}$, D. Elsaesser ${ }^{12}$, E. Farina ${ }^{17}$, D. Ferenc ${ }^{5}$, M. V. Fonseca ${ }^{7}$, L. Font ${ }^{18}$, K. Frantzen ${ }^{14}$, C. Fruck ${ }^{6}$, R. J. García López ${ }^{8}$, M. Garczarczyk ${ }^{10}$, D. Garrido Terrats ${ }^{18}$, M. Gaug ${ }^{18}$, G. Giavitto ${ }^{1}$, N. Godinović ${ }^{5}$, A. González Muñoz ${ }^{1}$, S. R. Gozzini ${ }^{10}$, A. Hadamek ${ }^{14}$, D. Hadasch ${ }^{19}$, A. Herrero ${ }^{8}$, D. Hildebrand ${ }^{11, \star}$, J. Hose $^{6}$, D. Hrupec ${ }^{5}$, W. Idec ${ }^{9}$, V. Kadenius ${ }^{20}$, H. Kellermann ${ }^{6}$, M. L. Knoetig ${ }^{11}$, J. Krause ${ }^{6}$, J. Kushida ${ }^{21}$, A. La Barbera ${ }^{3}$, D. Lelas ${ }^{5}$, N. Lewandowska ${ }^{12}$, E. Lindfors ${ }^{20,27, \star}$, S. Lombardi ${ }^{3, \star}$, M. López ${ }^{7}$, R. López-Coto ${ }^{1}$, A. López-Oramas ${ }^{1}$,

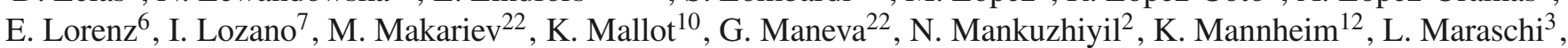
B. Marcote ${ }^{23}$, M. Mariotti ${ }^{16}$, M. Martínez ${ }^{1}$, D. Mazin ${ }^{6}$, U. Menzel $^{6}$, M. Meucci ${ }^{4}$, J. M. Miranda ${ }^{4}$, R. Mirzoyan ${ }^{6}$, A. Moralejo ${ }^{1}$, P. Munar-Adrover ${ }^{23}$, D. Nakajima ${ }^{21}$, A. Niedzwiecki ${ }^{9}$, K. Nilsson ${ }^{20,27}$, N. Nowak ${ }^{6}$, R. Orito ${ }^{21}$, A. Overkemping ${ }^{14}$, S. Paiano ${ }^{16}$, M. Palatiello ${ }^{2}$, D. Paneque ${ }^{6}$, R. Paoletti ${ }^{4}$, J. M. Paredes ${ }^{23}$, X. Paredes-Fortuny ${ }^{23}$, S. Partini ${ }^{4}$, M. Persic ${ }^{23}$, F. Prada ${ }^{15,28}$, P. G. Prada Moroni ${ }^{24}$, E. Prandini ${ }^{16}$, S. Preziuso ${ }^{4}$, I. Puljak ${ }^{5}$, R. Reinthal ${ }^{20}$, W. Rhode ${ }^{14}$, M. Ribó ${ }^{23}$, J. Rico ${ }^{1}$, J. Rodriguez Garcia ${ }^{6}$, S. Rügamer ${ }^{12}$, A. Saggion ${ }^{16}$, T. Saito ${ }^{21}$, K. Saito ${ }^{21}$, M. Salvati ${ }^{3}$, K. Satalecka ${ }^{7}$, V. Scalzotto ${ }^{16}$, V. Scapin ${ }^{7}$, C. Schultz ${ }^{16}$, T. Schweizer ${ }^{6}$, S. N. Shore ${ }^{24}$, A. Sillanpää ${ }^{20}$, J. Sitarek ${ }^{1}$, I. Snidaric ${ }^{5}$, D. Sobczynska ${ }^{9}$, F. Spanier ${ }^{12}$, V. Stamatescu ${ }^{1}$, A. Stamerra ${ }^{3}$, T. Steinbring ${ }^{12}$, J. Storz ${ }^{12}$, S. Sun ${ }^{6}$, T. Suric ${ }^{5}$,

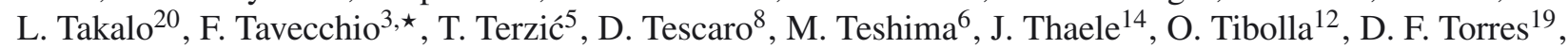
T. Toyama ${ }^{6}$, A. Treves ${ }^{17}$, M. Uellenbeck ${ }^{14}$, P. Vogler ${ }^{11}$, R. M. Wagner ${ }^{6,29}$, F. Zandanel $^{15,30}$, R. Zanin $^{23}$ (The MAGIC Collaboration), B. Balmaverde ${ }^{31}$, J. Kataoka ${ }^{32}$, R. Rekola ${ }^{20}$, and Y. Takahashi ${ }^{32}$

(Affiliations can be found after the references)

Received 28 October 2013 / Accepted 23 January 2014

\section{ABSTRACT}

Aims. The radio galaxy NGC 1275, recently identified as a very high energy (VHE, $>100 \mathrm{GeV}$ ) $\gamma$-ray emitter by MAGIC, is one of the few non-blazar active galactic nuclei detected in the VHE regime. The purpose of this work is to better understand the origin of the $\gamma$-ray emission and locate it within the galaxy.

Methods. We studied contemporaneous multifrequency observations of NGC 1275 and modeled the overall spectral energy distribution. We analyzed unpublished MAGIC observations carried out between October 2009 and February 2010, and the previously published observations taken between August 2010 and February 2011. We studied the multiband variability and correlations by analyzing data of Fermi-LAT in the $100 \mathrm{MeV}-100 \mathrm{GeV}$ energy band, as well as Chandra (X-ray), KVA (optical), and MOJAVE (radio) data taken during the same period. Results. Using customized Monte Carlo simulations corresponding to early MAGIC stereoscopic data, we detect NGC 1275 also in the earlier MAGIC campaign. The flux level and energy spectra are similar to the results of the second campaign. The monthly light curve above $100 \mathrm{GeV}$ shows a hint of variability at the $3.6 \sigma$ level. In the Fermi-LAT band, both flux and spectral shape variabilities are reported. The optical light curve is also variable and shows a clear correlation with the $\gamma$-ray flux above $100 \mathrm{MeV}$. In radio, three compact components are resolved in the innermost part of the jet. One of these components shows a similar trend as the Fermi-LAT and KVA light curves. The $\gamma$-ray spectra measured simultaneously with MAGIC and Fermi-LAT from $100 \mathrm{MeV}$ to $650 \mathrm{GeV}$ can be well fitted either by a log-parabola or by a power-law with a subexponential cutoff for the two observation campaigns. A single-zone synchrotron-self-Compton model, with an electron spectrum following a power-law with an exponential cutoff, can explain the broadband spectral energy distribution and the multifrequency behavior of the source. However, this model suggests an untypical low bulk-Lorentz factor or a velocity alignment closer to the line of sight than the parsec-scale radio jet.

Key words. galaxies: active - gamma rays: galaxies - galaxies: jets - galaxies: individual: NGC 1275

\section{Introduction}

^ Corresponding author: e-mail: colin@mppmu.mpg.de , saverio.lombardi@oa-roma.inaf.it, fabrizio.tavecchio@brera.inaf.it, dorothee.hildebrand@phys.ethz.ch, elilin@utu.fi
The radio galaxy NGC 1275 is the central dominant galaxy of the Perseus cluster (Abell 426). It is a well-known active galactic nucleus (AGN) already included in the original study of Seyfert (1943). In fact, it is a rather complex object with very 
peculiar characteristics that might be the result of massive gas accretion from the cluster cooling flow or from a recent merging event (Conselice et al. 2001). The main features, in addition to the central AGN, are remarkable gas filaments that are tens of kpc long and emit strong hydrogen lines (Lynds 1970), and a foreground galaxy that falls toward NGC 1275 (Caulet et al. 1992).

In radio, the emission is dominated by a very bright compact source $(3 \mathrm{C} 84)$ at the center of the galaxy. The core-dominated morphology with asymmetrical jets at both kpc (Pedlar et al. 1990) and pc scales (Asada et al. 2006) looks like a FanaroffRiley type I (FR I) radio galaxy with a jet axis relatively close to the line of sight. The faint counter jet measured with Very Long Baseline Interferometry (VLBI) suggests a jet angle of $30^{\circ}-55^{\circ}$ in the core region (Vermeulen et al. 1994; Walker et al. 1994; Asada et al. 2006). At larger scales, there is evidence of jet bending (Pedlar et al. 1990). The flux and morphology of the core radio emission are variable. Recent observations showed that an outburst is on-going since 2005 (Nagai et al. 2010). At subparsec scales, a new component appeared near the nucleus in 2007 and continues to grow in flux as it travels away from the nucleus. Since 2010, its brightness at $15 \mathrm{GHz}$ has exceeded that of the nucleus (Nagai et al. 2012).

The X-ray emission is dominated by the thermal emission of the intracluster medium cooling flow in the cluster central region (Churazov et al. 2003; Fabian et al. 2011). However, a nonthermal component from the nucleus is detected with highresolution instruments (Churazov et al. 2003; Balmaverde et al. 2006), which follows a hard power-law spectrum (photon index $\Gamma=1.6 \pm 0.1)$ in the $0.5-10 \mathrm{keV}$ range. Nonthermal emission also appears in hard X-rays above $\sim 20 \mathrm{keV}$ (Ajello et al. 2009; Eckert \& Paltini 2009), which matches the extrapolation of the AGN power-law X-ray emission well.

In $\gamma$-rays, emission from both the cluster and the AGN was expected. A first hint of high-energy emission from the NGC 1275 region was found in the COS B data [1975-1982] (Strong \& Bignami 1983) but was not confirmed by the next instrument CGRO-EGRET, which started observations one decade later [1991-2000] (Reimer at al. 2003). After the first four months of the all-sky survey with Fermi-LAT in 2008, $\gamma$-ray emission from NGC 1275 was clearly established (Abdo et al. 2009). The measured flux above $100 \mathrm{MeV}$ was seven times higher than the CGRO-EGRET upper limit, suggesting strong variability of the source. Subsequent Fermi-LAT observations confirmed this variability, revealing variation time-scales as rapid as a week (Kataoka et al. 2010; Brown \& Adams 2011).

At very high energies (VHE, >100 GeV), NGC 1275 has been observed without success by many Cherenkov telescope experiments: HEGRA (Goetting et al. 2001), Whipple$10 \mathrm{~m}$ (Perkins et al. 2006), VERITAS (Acciari et al. 2009), and MAGIC-I (Aleksić et al. 2010b). The first detection was recently reported by MAGIC in stereoscopic mode (Aleksić et al. 2012a). The 70-500 GeV energy spectrum is much steeper than the $0.1-$ $20 \mathrm{GeV}$ spectrum measured by Fermi-LAT, suggesting a break or a cutoff at a few tens of GeV. The angular resolution of the $\gamma$-ray telescopes is not sufficient to determine the origin of the emission within the galaxy, but the rapid variability seen by Fermi-LAT implies a very compact emission region, most likely from the inner part of the AGN. Additionally, the expected cluster $\gamma$-ray emission probably extends across a region of several hundred kpc (Pinzke \& Pfrommer 2010), and then must be constant on human time-scales. This emission may be detectable when the AGN is quiet (Colafrancesco et al. 2010) or above the high-energy cutoff of the AGN (Aleksić et al. 2012b).
In this work, we study contemporaneous multifrequency observations of the AGN emission. We analyzed the MAGIC data taken in stereoscopic mode from October 2009 to February 2011 as well as Fermi-LAT data, X-ray Chandra data, optical data of KVA and NOT, and radio data of the MOJAVE program at VLBA from the same observation period. Together with additional information from archival data, we discuss a possible emission scenario and try to model the source as a misaligned BL Lac object.

\section{Observations and data analysis}

\subsection{MAGIC}

MAGIC is a system of two imaging atmospheric Cherenkov telescopes $17 \mathrm{~m}$ in diameter located at the Roque de los Muchachos observatory $\left(28.8^{\circ} \mathrm{N}, 17.8^{\circ} \mathrm{W}, 2200 \mathrm{~m}\right.$ a.s.l. $)$, on the Canary Island of La Palma. The MAGIC telescopes have been operating in stereoscopic mode since October 2009 with a sensitivity $\leq 0.8 \%$ of the Crab Nebula flux, for energies above $\sim 300 \mathrm{GeV}$, in $50 \mathrm{~h}$ of observations (Aleksić et al. 2012c). The trigger system, optimized for the lowest energies, enables one to study $\gamma$-ray emission down to $50 \mathrm{GeV}$.

The MAGIC telescopes observed NGC 1275 during two distinct observational campaigns performed between October 2009 and February $2010(\sim 45.3 \mathrm{~h})$, and August 2010 and February 2011 ( $\sim 53.6 \mathrm{~h})$. Both campaigns were carried out in stereoscopic mode, but under different global trigger configurations.

The first campaign (Camp. 1) occurred partially during the commissioning phase of the second telescope (MAGIC-II). It was performed in the so-called soft-stereo trigger mode, with the first telescope (MAGIC-I) trigger working in single mode and the second telescope (MAGIC-II) recording only events triggered by both telescopes. This campaign resulted in the discovery of $\gamma$-ray emission above $300 \mathrm{GeV}$ from IC 310, another radio galaxy of the Perseus cluster (Aleksić et al. 2010a, 2014).

In the second campaign (Camp.2), observations were instead taken in the standard full-stereo trigger mode (recorded events are triggered simultaneously by both telescopes). It resulted in the first detection of NGC 1275 at energies above $100 \mathrm{GeV}$ (Aleksić et al. 2012a).

The soft-stereo trigger allows us to analyze MAGIC-I data in single mode but has a slightly higher energy threshold for stereoscopic data than the full-stereo trigger. However, above $\sim 150 \mathrm{GeV}$ data collected in both campaigns are almost identical and can be treated as a uniform data sample. This was the case for the study of the $\gamma$-ray emission induced by cosmic-ray population in the Perseus cluster (Aleksić et al. 2012b). Instead, for data analysis below $150 \mathrm{GeV}$ (as performed here), the two campaigns must be analyzed separately.

Both surveys were performed in the false-source tracking (wobble) mode (Fomin et al. 1994), with data equally split into two (Camp. 1) or four (Camp. 2) pointing positions located symmetrically at $0.4^{\circ}$ from NGC 1275 . Observations were carried out at low zenith angles $\left(<35^{\circ}\right)$, which resulted in an analysis energy threshold (defined as the peak of the $\gamma$-ray energy distribution for a Crab-like spectrum) of $100 \mathrm{GeV}$.

The data analysis was performed using the standard software package MARS (Aliu et al. 2009), which includes the latest standard routines for stereoscopic analysis (Aleksić et al. 2012c; Lombardi et al. 2011). The $\gamma$-ray selection cuts were optimized by means of contemporaneous Crab Nebula data and Monte Carlo simulations. The background was estimated from mirror regions (off-regions) corresponding to the source position 


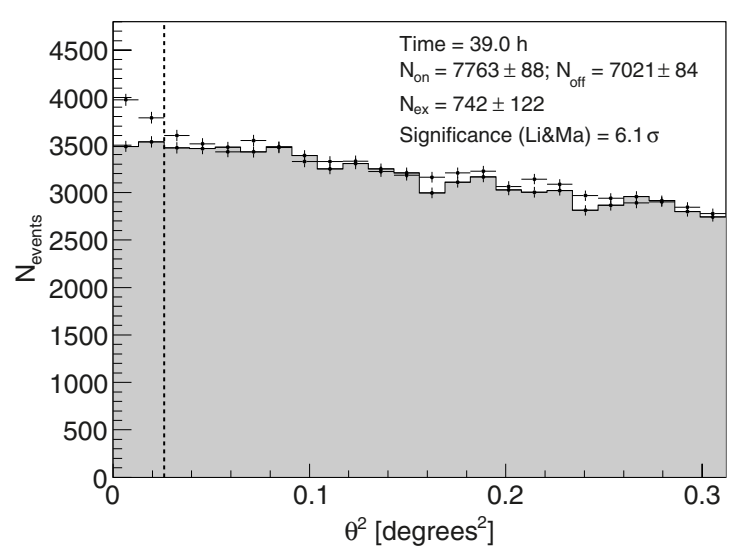

Fig. 1. $\theta^{2}$ distributions of the NGC 1275 signal and background estimation from $39.0 \mathrm{~h}$ of MAGIC stereo observations taken between October 2009 and February 2010, in soft-trigger stereo mode (see 2.1) with an energy threshold of $100 \mathrm{GeV}$. The region between zero and the vertical dashed line (at 0.026 degrees $^{2}$ ) represents the signal region.

in the camera during the other wobble pointing. Thus, depending on the number of wobble positions used in the campaign, a single off-region (Camp. 1) or three off-regions (Camp. 2) were considered. While the standard Monte Carlo simulations were used for the analysis of Camp. 2 data (reported in Aleksić et al. 2012a), the data analysis of the first campaign required new dedicated simulations to fully take into account the nonstandard trigger condition at the lowest energies $(<150 \mathrm{GeV})$.

After applying standard quality checks based on the rate of the stereo events and the distributions of basic image parameters, 39.0 h (Camp. 1) and 45.7 h (Camp. 2) of data were selected to derive the results presented here. The rejected data were affected mainly by poor atmospheric conditions during the data taking.

The $\gamma$-ray signal from the NGC 1275 is detected only at low energies. Figure 1 shows the $\theta^{2}$ plot $^{1}$ obtained with Camp. 1 data (October 2009-February 2010) for analysis cuts corresponding to an energy threshold of $100 \mathrm{GeV}$. We found an excess of $742 \pm 122$ events in the fiducial signal region with $\theta^{2}<0.026$ degree $^{2}$, corresponding to a significance of $6.1 \sigma$, calculated according to the Eq. (17) of Li \& Ma (1983). During the second campaign (August 2010-February 2011), an excess of $522 \pm 81$ events above the same energy threshold was detected with a significance of $6.6 \sigma$. Detailed Camp. 2 results are reported in Aleksić et al. (2012a).

The significance skymap above $100 \mathrm{GeV}$ for the first campaign is shown in Fig. 2. The central hot spot $(>6 \sigma)$ corresponding to the NGC 1275 position is consistent with a pointlike source. Conversely to the skymap above $400 \mathrm{GeV}$ reported in Aleksić et al. (2010a) for the same period, the radio galaxy IC 310 is not visible in the $\sim 100 \mathrm{GeV}$ skymap due to its very hard spectrum.

The unfolded differential energy spectra of the source derived from the two observational campaigns are shown in Fig. 3. The error bars represent the statistical uncertainties only, but for the power-law fit parameterization the systematic effects ${ }^{2}$

\footnotetext{
The parameter $\theta^{2}$ is the square angular distance between the reconstructed source of the events and the nominal positions of the expected source.

2 The systematic errors of the flux normalization and the energy spectral slope considered here have been estimated to be lower than $23 \%$ and \pm 0.3 , respectively, whereas the systematic uncertainty on the energy scale is $17 \%$. These values are more conservative than those presented
}

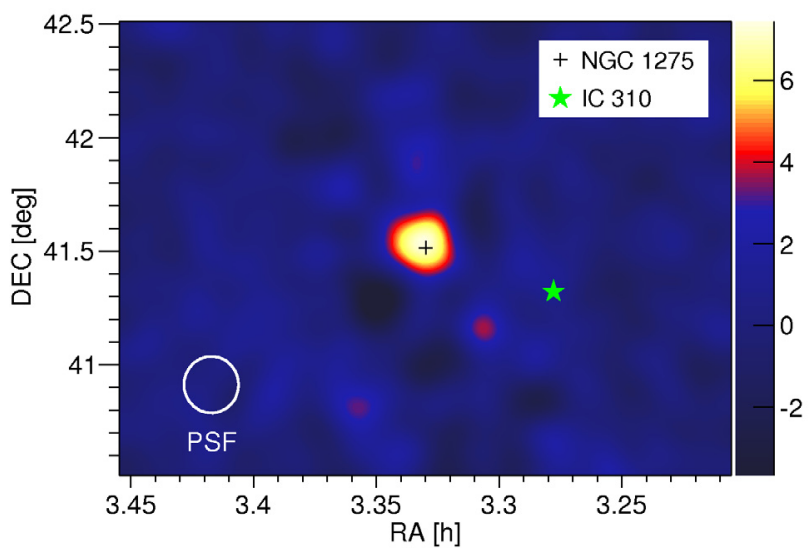

Fig. 2. Significance skymap (J2000) of the NGC 1275 region from $39.0 \mathrm{~h}$ of MAGIC stereo observations taken between October 2009 and February 2010, with a low energy threshold of $100 \mathrm{GeV}$. The point-spread function (PSF) of about $0.12^{\circ}$ is also displayed.

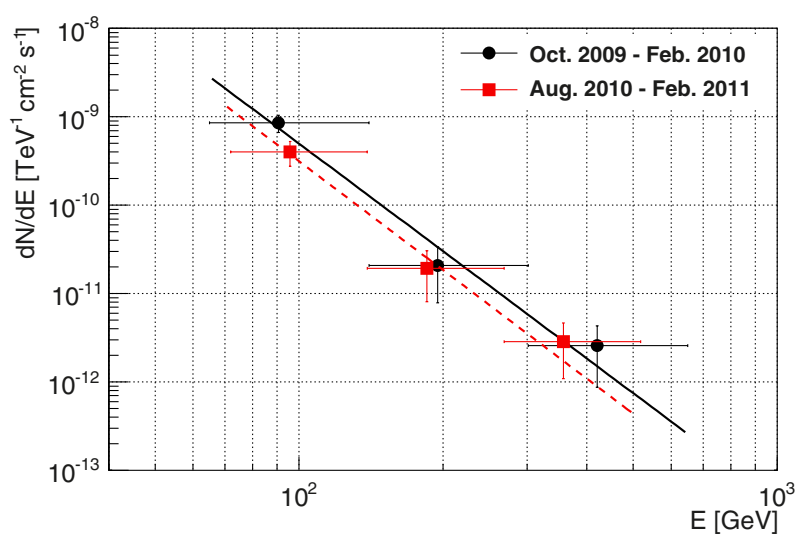

Fig. 3. NGC 1275 differential energy spectrum measured with MAGIC during two observation campaigns and their associated power-law fits. Camp. 1 data are in black with a solid line fit and Camp. 2 data are in red with a dashed line fit.

are also taken into account. For Camp. 1, the spectrum between $65 \mathrm{GeV}$ and $650 \mathrm{GeV}$ can be described by a simple power-law $\left(\chi^{2} / n_{\text {d.o.f. }}=4.86 / 3\right)$

$$
\frac{\mathrm{d} F}{\mathrm{~d} E}=f_{0}\left(\frac{E}{100 \mathrm{GeV}}\right)^{-\Gamma},
$$

with a photon index $\Gamma=4.0 \pm 0.5_{\text {stat }} \pm 0.3_{\text {syst }}$ and a normalization constant at $100 \mathrm{GeV} f_{0}=\left(5.0 \pm 0.9_{\text {stat }} \pm 1.1_{\text {syst }}\right) \times$ $10^{-10} \mathrm{~cm}^{-2} \mathrm{~s}^{-1} \mathrm{TeV}^{-1}$. The spectrum of Camp. 2 corresponds to the result reported in Aleksić et al. (2012a): $f_{0}=\left(3.1 \pm 1.0_{\text {stat }} \pm\right.$ $\left.0.7_{\text {syst }}\right) \times 10^{-10} \mathrm{~cm}^{-2} \mathrm{~s}^{-1} \mathrm{TeV}^{-1}$ and $\Gamma=4.1 \pm 0.7_{\text {stat }} \pm 0.3_{\text {syst }}$. The energy range of the Camp. 1 spectrum is slightly larger than that of Camp. 2. This arises because the Camp. 1 spectrum must have slightly larger bin widths in energy to fulfill the significance requirement of each bin.

The mean flux above $100 \mathrm{GeV}$ during Camp. 1 , (1.6 \pm $\left.0.3_{\text {stat }} \pm 0.3_{\text {syst }}\right) \times 10^{-11} \mathrm{~cm}^{-2} \mathrm{~s}^{-1}$, corresponds to about $3 \%$ of the Crab nebula flux. It is slightly higher than the Camp. 2 results, $\left(1.3 \pm 0.2_{\text {stat }} \pm 0.3_{\text {syst }}\right) \times 10^{-11} \mathrm{~cm}^{-2} \mathrm{~s}^{-1}$, but both campaigns agree within the uncertainties. No indication of spectral index variability has been found between the two campaigns. The monthly

in Aleksic et al. (2012c), because of the flux weakness and the spectral steepness of NGC 1275, as measured by MAGIC. 

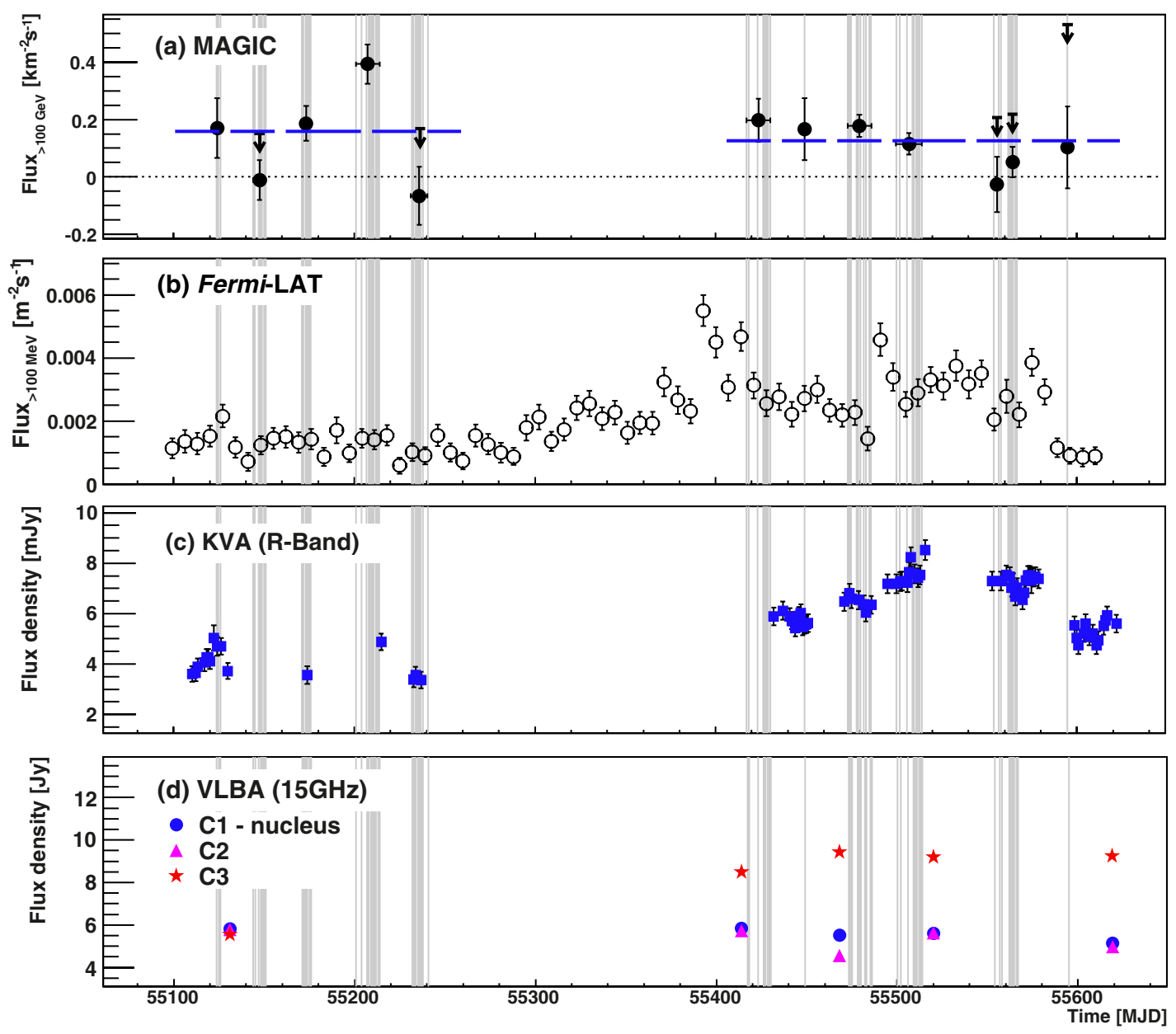

Fig. 4. Multifrequency light curves of NGC 1275 from October 2009 to February 2011. The vertical gray lines show the exact observation dates with MAGIC. a) The MAGIC LC above $100 \mathrm{GeV}$ in monthly bins. The thick dashed lines represent the constant fit for both observation campaigns. For data showing an excess $<1 \sigma$, upper limits (black arrows) are calculated assuming a spectral index $\Gamma=4.0$, using the Rolke et al. 2005 method with a confidence level of $95 \%$ and a total systematic uncertainty of $30 \%$. b) The Fermi-LAT LC above $100 \mathrm{MeV}$ has a weekly binning. c) The KVA $R$-band LC shows the core continuum flux corrected for Galactic extinction. The constant systematic error $( \pm 0.6 \mathrm{mJy})$ induced by the hostgalaxy and emission-line subtraction is not included in the error bars. d) The VLBA LCs show the $15 \mathrm{GHz}$ emission from the three innermost components of the radio jet.

light curve (LC) of NGC 1275 above $100 \mathrm{GeV}$ is shown in the top panel of Fig. 4. While the Camp. 2 LC is quite consistent with a constant flux $\left(\chi^{2} / n_{\text {d.o.f. }}=7.4 / 4\right.$, probability $\left.=0.29\right)$, a hint of variability can be derived for the first campaign. Indeed, fitting the Camp. $1 \mathrm{LC}$ with a constant flux yields a $\chi^{2} / n_{\text {d.o.f. }}=22.9 / 4$ (probability $=1.3 \times 10^{-4}$ ), corresponding to a significance for a monthly variable emission of $3.6 \sigma$.

\subsection{Fermi-LAT}

The Large Area Telescope (LAT) is a pair-conversion telescope onboard the Fermi $\gamma$-ray Space Telescope, designed to cover the energy band from $20 \mathrm{MeV}$ to higher than $300 \mathrm{GeV}$ (Atwood et al. 2009). The observations used here comprise all scientific data obtained between August 4, 2008 and February 21, 2011. We applied a zenith angle cut of $100^{\circ}$ to greatly reduce $\gamma$-rays from Earth's limb. The same zenith cut is also accounted for in the exposure calculation using the LAT Science Tool gtltcube. We used the "Source" class events (Ackermann et al. 2012), which is the recommended class for regular analysis. In our analysis, Science Tools version v9r27p1 and instrumental response functions P7SOURCE_V6 were used. The lower energy bound was set at $100 \mathrm{MeV}$ and the region of interest (ROI) radius at $10^{\circ}$ (see Abdo et al. 2009). All the nearby sources in the 2FGL catalog (Nolan et al. 2012) were included in the model of the ROI, fixing their spectral index and letting their normalization free in the fit. Since no variability is expected for the underlying background diffuse emission, we fixed the normalization of both Galactic and extragalactic diffuse backgrounds to the average values determined from the whole observing period described in this paper (from August 4, 2008 to February 21, 2011). The early portion of the data between August 4, 2008 and August 13, 2009 coincides with the early LAT observations of NGC 1275 presented in Kataoka et al. (2010; see also Brown \& Adams 2011). The systematic uncertainties on the flux were estimated as $10 \%$ at $100 \mathrm{MeV}$, decreasing to $5 \%$ at $560 \mathrm{MeV}$, and increasing to $10 \%$ at $10 \mathrm{GeV}$ and higher (Ackermann et al. 2012).

Panel (b) of Fig. 4 shows the $\gamma$-ray flux above $100 \mathrm{MeV}$ of NGC 1275 from September 25, 2009 to February 18, 2011 binned at one-week intervals. In each bin, the significance of the NGC 1275 detection is higher than the test statistic $T S>10$ and the ratio of flux error to flux is below $0.5(\delta F / F \lesssim 0.5$, see Nolan et al. 2012). There is a general trend of increasing flux toward the end of the observations, and several episodes of large flares with a flux variability as fast as one week. 


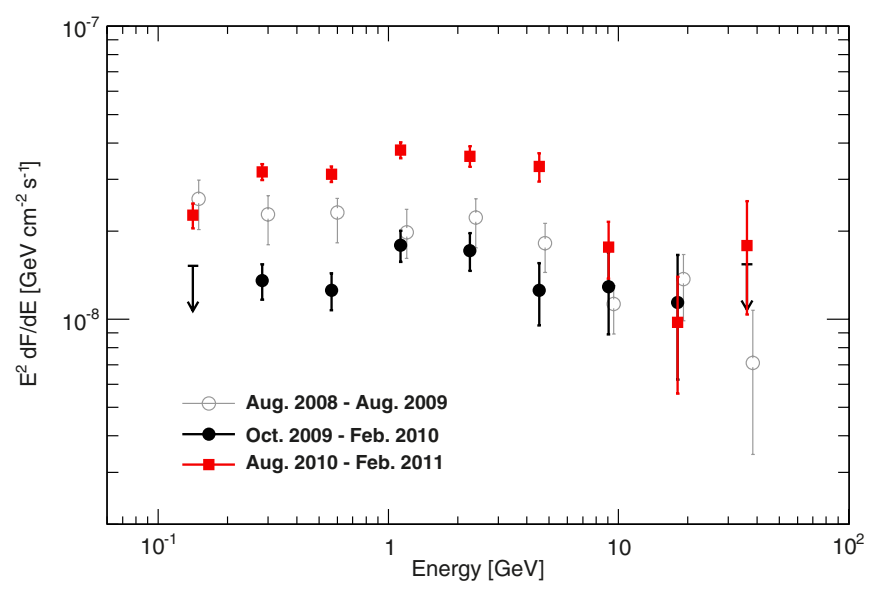

Fig. 5. NGC 1275 SED measured with Fermi-LAT during several periods corresponding to the first year of operation (empty circles, Kataoka et al. 2010), the first MAGIC campaign (full circles and black arrows), and the second MAGIC campaign (full squares).

Figure 5 shows the Fermi-LAT spectral energy distributions (SED) derived for the two MAGIC-observation campaigns (MJD 55 123-55 241 for Camp. 1 and MJD 55417-55 595 for Camp. 2) compared with that obtained for the first year given in Kataoka et al. (2010). The spectra were obtained from nine independent energy bins: $(0.1-0.2,0.2-0.4,0.4-0.8,0.8-1.6$, 1.6-3.2, 3.2-6.4, 6.4-12.8, 12.8-25.6, and 25.6-51.2) GeV. The general spectral shape can be approximated by a power-law function with a photon index $\Gamma \simeq 2$, slightly curving down toward the highest energies. Substantial spectral evolution can be seen in different observational periods, both in the flux and peak frequencies of the $\gamma$-ray emissions. The Camp. 2 mean GeV flux is about twice as high as during the first MAGIC campaign, and the Camp. 2 SED shows a clear curvature peaking around $1 \mathrm{GeV}$, whereas the first-year SED is constantly decreasing with energy. However, above a few $\mathrm{GeV}$ all the spectra reach similar fluxes. The $\gamma$-ray flux seems more variable at low energy. The precise fit characterization of the LAT spectrum is discussed together with the MAGIC data in Sect. 3.3.

\subsection{Chandra X-ray Observatory}

The ACIS detector onboard the Chandra X-ray Observatory is a spectrometer with very high angular resolution $\left(\sim 0.5^{\prime \prime}\right)$ operating in the range of $0.2-10 \mathrm{keV}$. In the public archive, we found seven observations performed during the MAGIC observing period (namely ObsId 11713, 11714, 11715, 12025, 12033, 12036, and 12037 ; PI Fabian). In NGC 1275, the nuclear X-ray flux is high enough that pileup is potentially a severe problem. For an on-axis observation, PIMMS estimates a total pileup fraction of about $52 \%$ (i.e. more than half of the incident photons are either combined into piled events or removed because their energy is higher than the spacecraft threshold). The nucleus was not the primary target of these observations and no observing strategy for minimizing nuclear pileup was actuated (e.g. selecting a subarray to reduce the nominal frame integration time). However, this set of observations was centered several arc-minutes away from the nucleus $\left(3.2^{\prime}-7.6^{\prime}\right)$, and this reduces the pileup because at larger offset the effective area of the mirrors is lower and the point-spread function (PSF) is larger. We analyzed the three observations with offset angles $>7.5^{\prime}$ (ObsId 12025, 12033, 12036) that are less affected by the pileup. The measured counts to frame are still higher than the typical threshold $(0.1-0.2$, e.g.
Table 1. Unabsorbed integral flux of the NGC 1275 nonthermal powerlaw core emission in the range $2-10 \mathrm{keV}$ obtained from Chandra observations assuming different power-law slopes.

\begin{tabular}{ccccc}
\hline \hline ObsId & Date & $\Gamma$ & fit $\chi^{2}$ (d.o.f.) & $F(2-10 \mathrm{keV})^{a}$ \\
\hline 12025 & $2009-11-25$ & & & $1.26_{-0.1}^{+0.1}$ \\
12033 & $2009-11-27$ & 2.5 & $1616.8(1075)$ & $1.27_{-0.1}^{+0.1}$ \\
12036 & $2009-12-02$ & & & $1.35_{-0.1}^{+0.1}$ \\
\hline 12025 & $2009-11-25$ & & & $1.50_{-0.1}^{+0.1}$ \\
12033 & $2009-11-27$ & 2.0 & $1307.4(1075)$ & $1.49_{-0.1}^{+0.5}$ \\
12036 & $2009-12-02$ & & & $1.71_{-0.2}^{+0.4}$ \\
\hline 12025 & $2009-11-25$ & & & $2.20_{-0.3}^{+0.3}$ \\
12033 & $2009-11-27$ & 1.7 & $1392.8(1075)$ & $4.40_{-0.3}^{+1.2}$ \\
12036 & $2009-12-02$ & & & $5.60_{-0.2}^{+0.1}$ \\
\hline
\end{tabular}

Notes. ${ }^{(a)}$ Integral flux in $10^{-11} \mathrm{erg} \mathrm{cm}^{-2} \mathrm{~s}^{-1}$ (uncertainties at $\pm 1 \sigma$ ).

Massaro et al. 2012) above which the pileup is a problem. Thus, a pileup model (Davis 2001) is statistically required to fit the data.

We applied the standard data reduction procedure ${ }^{3}$ using the Chandra Interactive Analysis of Observations CIAO 4.4, with Chandra Calibration Database CAL-DB version 4.4.1. We generated a new level $=2$ event file by applying the standard grade, status, and good time filters. We rebinned the spectrum using a 25-count threshold and fitted the spectrum in the range 0.5$9.5 \mathrm{keV}$. The three spectra were fitted simultaneously by adopting the model pileup $\times$ phabs $($ mekal + zphabs (powerlaw) $)$ in XSPEC version: 12.7.1, where mekal models the thermal and line emissions of the hot diffuse gas, powerlaw is the nonthermal power-law emission from the jet, pileup reproduces the pileup effect and, phabs and zphabs correspond to the Galactic and internal absorptions. The metal abundance and hydrogen density of mekal were fixed to 0.7 solar value and $0.1 \mathrm{~cm}^{-3}$, respectively. The temperature and normalization are obtained from the fit $(k T=0.30 \pm 0.01 \mathrm{keV})$. For the absorption, we fixed the hydrogen column density from the Galaxy $\left(N_{\mathrm{H}, \mathrm{gal}}=\right.$ $\left.1.5 \times 10^{21} \mathrm{~cm}^{-2}\right)$ and let free the internal absorption $\left(z N_{\mathrm{H}}=\right.$ $5.6 \pm 0.2 \times 10^{21} \mathrm{~cm}^{-2}$ ).

The power-law slope $\Gamma$ is strongly affected by the pileup effect, which reduces the counts at lower energy (because two or more photons are counted as one) and increases the counts at high energy. This creates a kind of degeneracy, and we decided to fix the parameter $\Gamma$ in the model. All the parameters of the powerlaw and mekal models were linked, except for the normalization. Table 1 provides the results of the fit for several powerlaw slope assumptions and Fig. 6 shows the measured energy spectra and the fit models for $\Gamma=2.5$.

\subsection{KVA and NOT}

NGC 1275 has been monitored in the optical $R$-band by the Tuorla blazar monitoring program ${ }^{4}$ since October 2009. The observations are performed using the KVA $35 \mathrm{~cm}$ telescope located at La Palma. The data were reduced using the standard data analysis pipeline and the magnitudes were measured with differential photometry with an aperture of 5.0" and the comparison stars from Fiorucci et al. (1998). The observed magnitudes varied

\footnotetext{
3 http://cxc.harvard.edu/ciao/guides/acis_data.html

4 http://users.utu.fi/kani/1m
} 

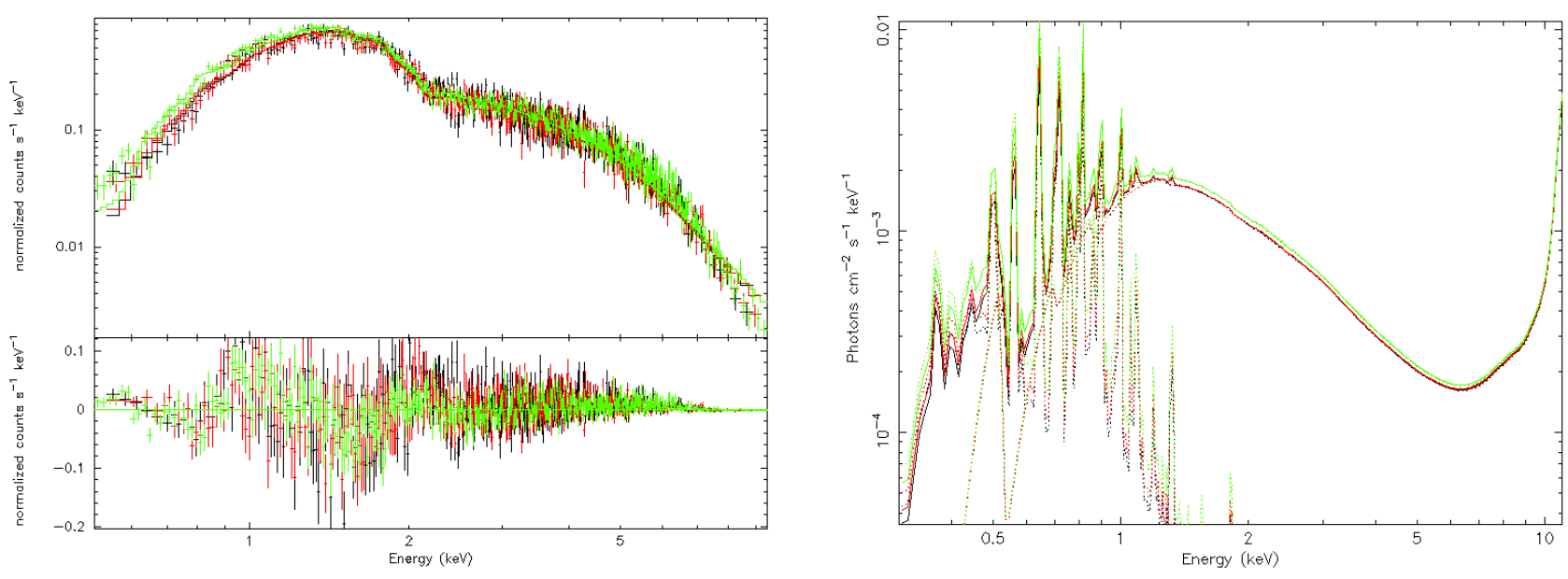

Fig. 6. Energy spectra of the NGC 1275 nuclear emission from the three large offset Chandra observations (ObsId 11025-green, 12033-red, 12036black) and the fit models with fixed power-law index $\Gamma=2.5$. In the top left panel, crosses show the photon rates per energy bin grouped to have at least 25 counts/bin and the solid lines show the fit models. In the bottom left panel, the fit residuals are reported. The right-hand panel shows the photon flux energy spectra of the fit models. Dashed lines represent the different model components (mekal and powerlaw after absorption and pileup effect) and the solid lines are the total. The large tail at high energy is a consequence of the pileup modeling.

between 13.05 and 13.3. The magnitudes were converted to flux using the standard formula $S=S_{0} \cdot 10^{-\operatorname{mag} / 2.5}$ and $S_{0}=3080 \mathrm{Jy}$.

To calculate the intrinsic emission from the core, the measured magnitudes over the MAGIC observing periods have to be averaged, the host galaxy subtracted, and the resulting data de-reddened. Additionally, the core flux is contaminated by emission lines that also need to be subtracted. To estimate the contributions of the host galaxy and the emission lines to the raw KVA measurements, NGC 1275 was observed with the Nordic Optical Telescope (NOT) on November 15, 2011 using the ALFOSC instrument. Five $60 \mathrm{~s} R$-band exposures and two spectra using grism 4 and slit 1 " were obtained. The images were reduced and combined using the standard IRAF tasks. The methodology of Nilsson et al. (2007) was used to estimate the contribution of the host galaxy. In short, the method is based on fitting a two-dimensional nucleus plus host galaxy model to the image and convolving the model with the PSF derived from the stars in the same field of view. The resulting host galaxy flux based on the aperture used is $11.08 \pm 0.55 \mathrm{mJy}$. The line contamination is estimated from the spectrum of NGC 1275 by integrating the observed line flux within the NOT $R$-band filter. We find that the line contamination in the $R$-band is $0.9 \mathrm{mJy}$, that is only $\sim 10 \%$ of the host flux. The host and line contaminations were then subtracted to derive the core continuum nonthermal flux, which was corrected for Galactic extinction (de-reddened) using the extinction value from Schlegel et al. (1998). The measured fluxes are shown together with the multifrequency light curves (Fig. 4). The mean fluxes over the two MAGIC campaigns are $(4.1 \pm 0.6) \mathrm{mJy}$ for Camp. 1 and $(6.5 \pm 0.6) \mathrm{mJy}$ for Camp. 2 .

\subsection{MOJAVE observations}

The Very Long Baseline Array (VLBA) is a radio interferometer using ten antennas $25 \mathrm{~m}$ in diameter located across the USA between Hawaii and the Virgin Islands. Thanks to its very long baselines (up to $8600 \mathrm{~km}$ ), angular resolutions down to milliarcseconds (mas) can be achieved. The Monitoring Of Jets in AGNs with VLBA Experiments (MOJAVE) program is constantly observing a set of bright AGNs in the northern hemisphere at $15 \mathrm{GHz}$ (Lister et al. 2009).
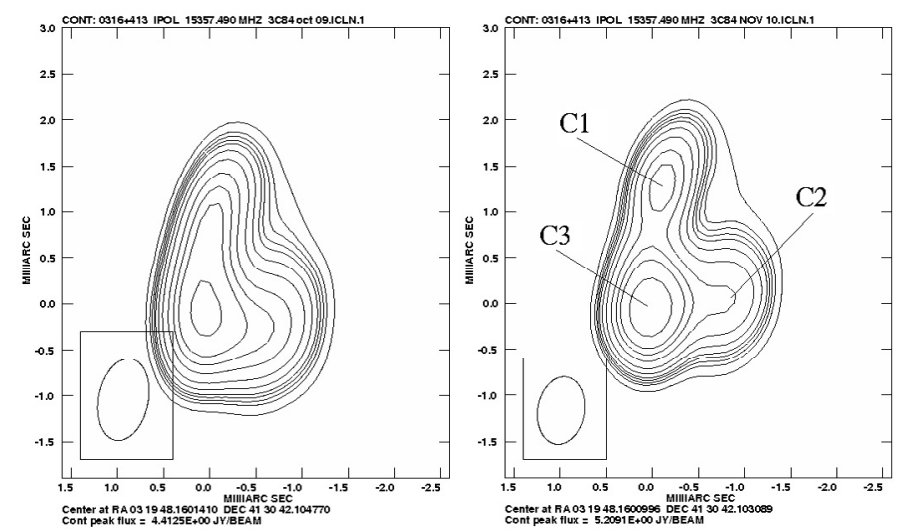

Fig. 7. MOJAVE images of $3 \mathrm{C} 84$ at $15 \mathrm{GHz}$ in October 2009 (left) and November 2010 (right). The contours are plotted at levels of $(0.5,0.7$, $0.8,0.9,1,1.2,1.5,2,2.5,3$, and 4) Jy/beam. The labels C1, C2, and C3 show the three bright components in the central region. The insets show the shape of the PSF.

The calibrated data of the MOJAVE program were reduced using the NRAO Astronomical Imaging Processing System (AIPS). In this work, we analyzed five epochs covering the time interval from October 2009 to February 2011. Figure 7 shows the total intensity images of the central region $(\sim 1 \mathrm{pc})$ at $15 \mathrm{GHz}$, featuring three bright components $\mathrm{C} 1, \mathrm{C} 2$, and $\mathrm{C} 3$. Generally $\mathrm{C} 1$ is understood to be the nuclear core emission. $\mathrm{C} 3$ corresponds to the rapidly growing component that recently appeared (Nagai et al. 2010). The position and flux density for each component was derived by means of the task JMFIT, which fits a Gaussian function to a selected area in the image plane. The obtained flux densities are listed in Table 2 and are shown in panel (d) of Fig. 4. We consider the VLBA accuracy of amplitude calibration as the dominant uncertainty (at the level of 5\%). While the flux densities of $\mathrm{C} 1$ and $\mathrm{C} 2$ do not show considerable variability, the intensity of $\mathrm{C} 3$ significantly increases between the beginning and the end of the observational campaign (as reported Table 2). 
Table 2. Flux densities of components $\mathrm{C} 1, \mathrm{C} 2$, and $\mathrm{C} 3$ obtained from MOJAVE data at $15 \mathrm{GHz}$.

\begin{tabular}{lccc}
\hline \hline Epoch & C1 (Jy) & C2 (Jy) & C3 (Jy) \\
\hline $2009-10-09$ & $5.80 \pm 0.29$ & $5.79 \pm 0.29$ & $5.54 \pm 0.27$ \\
$2010-08-06$ & $5.84 \pm 0.29$ & $5.74 \pm 0.29$ & $8.50 \pm 0.43$ \\
$2010-09-29$ & $5.48 \pm 0.27$ & $4.53 \pm 0.23$ & $9.42 \pm 0.47$ \\
$2010-11-20$ & $5.59 \pm 0.28$ & $5.62 \pm 0.28$ & $9.20 \pm 0.46$ \\
$2011-02-27$ & $5.13 \pm 0.26$ & $4.97 \pm 0.25$ & $9.21 \pm 0.46$ \\
\hline
\end{tabular}

\subsection{Other multifrequency data}

NGC 1275 is a famous and bright object that is regularly observed at many frequencies. Some observations made during the period of interest here (October 2009-February 2011) were reported in scientific journals and can be used for our broadband study without dedicated data processing.

NGC 1275 was part of the Early Release Compact Source Catalogue of the Planck microwave observatory (Planck Collaboration 2011, and references therein). This catalog contains NGC 1275 observations taken during two periods: from August 29 to September 4, 2009 and from February 10 to 19, 2010. Both periods are close to the first MAGIC observation campaign. More recent Planck observations taken from August 29 to September 4, 2010, corresponding to the second MAGIC campaign, are reported in Giommi et al. (2012). The derived spectrum between $30 \mathrm{GHz}$ and $857 \mathrm{GHz}$ agrees well with the one reported in the Early Release Compact Source Catalogue between $30 \mathrm{GHz}$ and $353 \mathrm{GHz}$. The microwave flux of NGC 1275 was quite stable during the MAGIC observations.

Giommi et al. (2012) also reported contemporaneous observations in the ultraviolet (UV)/optical band (170-650 nm) with Swift-UVOT taken August 9, 2010 at the beginning of the second MAGIC campaign. Swift-XRT cannot easily be used to estimate the nuclear X-ray emission because its angular resolution does not allow isolation of the AGN from diffuse Perseus cluster central emission. The analysis of Swift-XRT and -BAT data reported in Ajello et al. (2009) shows that the cluster emission dominates until $\sim 30 \mathrm{keV}$. NGC $1275 \mathrm{X}$-ray emission is also monitored with Swift-BAT ${ }^{5}$. The light curve (dominated by the cluster emission) does not show any enhancement during the MAGIC observation period, suggesting that no exceptional hard X-ray flare occurred.

In radio, NGC 1275 was regularly monitored with the Metsähovi $14 \mathrm{~m}$ single-dish telescope with additional VLBI observations of the VERA stations at $43 \mathrm{GHz}$ (Nagai et al. 2012) and the MOJAVE program at $15 \mathrm{GHz}$ (discussed in Sect. 2.5). Moreover, the GENJI program (Nagai et al. 2013) started a dense VLBI monitoring of NGC 1275 at $22 \mathrm{GHz}$ in November 2010 (during the second MAGIC observation campaign). All radio data show the same trend of a flux slowly increasing with time from the component $\mathrm{C} 3$ during the period of interest.

\section{Interpretations}

\subsection{Data contamination}

The majority of known VHE $\gamma$-ray emitting AGNs are blazars, that is, their jet points directly to the observer. In these cases, the nonthermal jet emission is highly boosted and dominates other

\footnotetext{
http://swift.gsfc.nasa.gov/results/bs70mon/ SWIFT J0319.7p4132
}

components. It is rather simple to subtract the background emission from the host galaxy. For NGC 1275, however, this is much more difficult. Thermal and nonthermal radiations can be emitted by extended structures in the host galaxy or even the host cluster of galaxies. Thus, observations with different angular resolution can integrate signal from different regions.

While the rapid and strong variability measured by FermiLAT implies that the $\gamma$-ray emission is largely dominated by a compact source most likely close to the nucleus, there are strong indications that at other frequencies other emitting components contribute significantly to the observed flux. In optical, the KVA measurements were corrected for both the host-galaxy and emission-line contributions. They are still significantly higher than previous Hubble Space Telescope (HST) measurements taken in 1994-1995 (Chiaberge et al. 1999; Baldi et al. 2010) when the radio core emission was similar to its level in 20092011 (see Effelsberg light curve in Nagai et al. 2010). The HST observations are characterized by an extremely good angular resolution, allowing the emission to be pinpointed from the innermost regions of the jet. The small discrepancy between our measurement and the HST results might be explained by temporal variability, but also by the limited angular resolution of KVA, which may contain large-scale jet contributions. In radio, VLBA provides an extremely good angular resolution which allows us to resolve three components in the subparsec core region. However, this resolution $(\sim 0.2 \mathrm{pc})$ is still insufficient to probe the $\gamma$-ray emission-region size expected from the week-scale variability $(\sim 0.01 \mathrm{pc})$. Substructures not resolved by VLBA can therefore exist. Planck and UVOT both have relatively broad angular resolution and their measurements are most likely contaminated by large-scale emission. Thus our results from radio to $\mathrm{X}$-rays should be considered firstly as upper limits of the lowenergy counterpart of the $\gamma$-ray emitting region.

\subsection{Optical $\gamma$-ray correlation}

We measured flux variability at different energy bands (Fig. 4). The light curves for $\mathrm{GeV} \gamma$-rays, optical, and the $\mathrm{C} 3$ radio component all show a similar trend. The intense monitoring in optical and $\mathrm{GeV} \gamma$-rays allows us to study this correlation more deeply. For every time bin of the Fermi-LAT weekly LC containing simultaneous KVA observations, we calculated the mean optical flux density of the core. Figure 8 shows the $\gamma$-ray flux above $100 \mathrm{MeV}$ as a function of the optical core emission for the 23 independent weeks with simultaneous Fermi-LAT and KVA observations. A clear correlation is visible. The linear correlation coefficient (Bravais-Pearson coefficient) is $0.79_{-0.10}^{+0.07}$. The correlation can also be characterized using the prescription of Edelson \& Krolik (1988), which, unlike the Pearson coefficient calculation, takes into account the measurement uncertainties. This leads to a discrete correlation function $\mathrm{DCF}=0.86 \pm 0.21$. Both statistical procedures for quantifying the optical $\gamma$-ray correlation lead to the same conclusion: a positive correlation at the level of $4-5 \sigma$.

This correlation strongly suggests that the corrected KVA results are dominated by the optical counterpart of the $\gamma$-rayemitting region. The lower optical level measured in 1994-1995 by HST $(<2 \mathrm{mJy}$ ) is most likely due to a weaker inner jet activity which, indeed, was not detected in $\gamma$-rays with CGRO-EGRET during this period.

Different types of correlation can be expected depending on the emission model and on the physical parameter driving the flux variation. In a simple SSC model (see description Sect. 3.4), the variation of the electron density implies a 


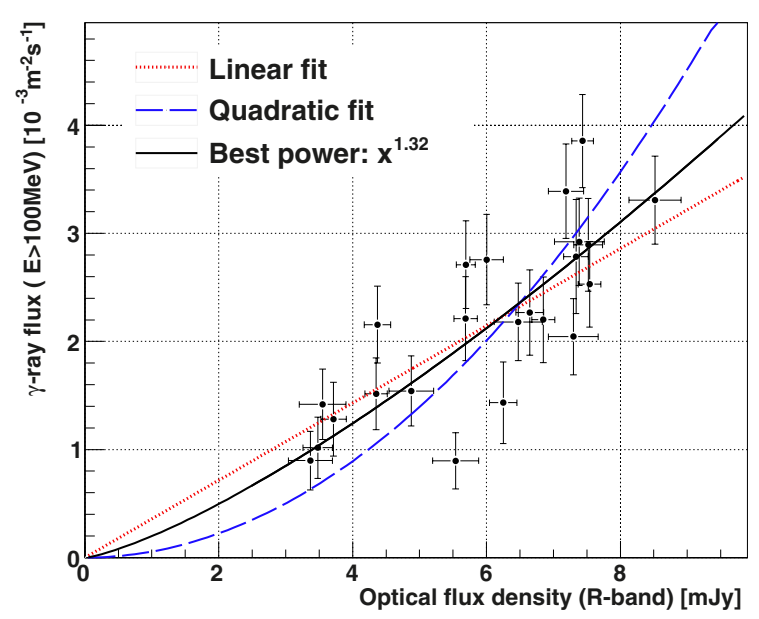

Fig. 8. NGC $1275 \gamma$-ray flux above $100 \mathrm{MeV}$ (Fermi-LAT) as a function of the optical continuum core flux density (KVA). The dotted and dashed lines represent the linear and quadratic fit, respectively. The solid line is the best fit with a free correlation factor $\left(F_{\gamma}=\alpha\left(F_{\text {opt }}\right)^{\beta}\right.$ with $\beta=1.32$ ).

Table 3. Parameters of the NGC $1275 \gamma$-ray SED fit over the energy range $0.1-650 \mathrm{GeV}$, for different fit functions.

\begin{tabular}{|c|c|c|c|c|c|}
\hline \multicolumn{5}{|c|}{ Power law: $\mathrm{d} F / \mathrm{d} E=f_{0}\left(\frac{E}{\mathrm{GeV}}\right)^{-\Gamma}$} & \\
\hline Epoch & $f_{0}{ }^{a}$ & $\Gamma$ & $\chi^{2} /$ d.o.f. & Prob. & \\
\hline Camp. 1 & $111 \pm 8$ & $2.38 \pm 0.03$ & $57.4 / 8$ & $1.5 \mathrm{e}-9$ & \\
\hline Camp. 2 & $176 \pm 7$ & $2.41 \pm 0.02$ & $317 / 10$ & $3 . e-62$ & \\
\hline \multicolumn{6}{|c|}{ Power law with exponential cutoff: $\frac{\mathrm{d} F}{\mathrm{~d} E}=f_{0}\left(\frac{E}{\mathrm{GeV}}\right)^{-\Gamma} \mathrm{e}^{-E / E_{\mathrm{c}}}$} \\
\hline Epoch & $f_{0}{ }^{a}$ & $\Gamma$ & $E_{\mathrm{c}}{ }^{b}$ & $\chi^{2} /$ d.o.f. & Prob. \\
\hline Camp. 1 & $149 \pm 10$ & $1.93 \pm 0.06$ & $67 \pm 12$ & $8.6 / 7$ & 0.28 \\
\hline Camp. 2 & $411 \pm 25$ & $1.75 \pm 0.05$ & $7.3 \pm 1.6$ & $26.5 / 9$ & 0.0017 \\
\hline \multicolumn{6}{|c|}{ Power law with a subexp. cutoff: $\frac{\mathrm{d} F}{\mathrm{~d} E}=f_{0}\left(\frac{E}{\mathrm{GeV}}\right)^{-\Gamma} \mathrm{e}^{-\sqrt{E / E_{\mathrm{c}}}}$} \\
\hline Epoch & $f_{0}^{a}$ & $\Gamma$ & $E_{\mathrm{c}}{ }^{b}$ & $\chi^{2} /$ d.o.f. & Prob. \\
\hline Camp. 1 & $201 \pm 17$ & $1.77 \pm 0.08$ & $13.1 \pm 4.2$ & $9.4 / 7$ & 0.22 \\
\hline Camp. 2 & $536 \pm 51$ & $1.72 \pm 0.05$ & $5.0 \pm 1.6$ & $19.3 / 9$ & 0.023 \\
\hline \multicolumn{6}{|c|}{ Log parabola: $\mathrm{d} F / \mathrm{d} E=f_{0}\left(\frac{E}{\mathrm{GeV}}\right)^{-2-\beta \log \left(E / E_{\mathrm{p}}\right)}$} \\
\hline Epoch & $f_{0}{ }^{a}$ & $\beta$ & $E_{\mathrm{p}}{ }^{c}$ & $\chi^{2} /$ d.o.f. & Prob. \\
\hline Camp. 1 & $163 \pm 11$ & $0.26 \pm 0.04$ & $2.8 \pm 1.5$ & $12.3 / 7$ & 0.09 \\
\hline Camp. 2 & $371 \pm 13$ & $0.31 \pm 0.03$ & $1.1 \pm 0.2$ & $13.4 / 9$ & 0.15 \\
\hline
\end{tabular}

Notes. ${ }^{(a)}$ Differential flux normalization in $10^{-10} \mathrm{GeV}^{-1} \mathrm{~cm}^{-2} \mathrm{~s}^{-1}$. ${ }^{(b)}$ Cutoff energy in GeV. ${ }^{(c)}$ Peak energy in GeV.

quadratic correlation, whereas the magnetic field variation implies a linear correlation. We fitted the data assuming correlation functions of the form $F_{\gamma}=\alpha\left(F_{\text {opt }}\right)^{\beta}$. The best fit is obtained for $\beta=1.32$, but both a linear $(\beta=1)$ and a quadratic $(\beta=2)$ correlation can fit the data as well.

\subsection{Fit of $\gamma$-ray SED}

The photon indices of the energy spectrum measured quasisimultaneously with Fermi-LAT $(\Gamma \simeq 2)$ and MAGIC $(\Gamma \simeq 4)$ are very different. Figure 9 shows the SED between $100 \mathrm{MeV}$ and $650 \mathrm{GeV}$ corresponding to the two observation campaigns.
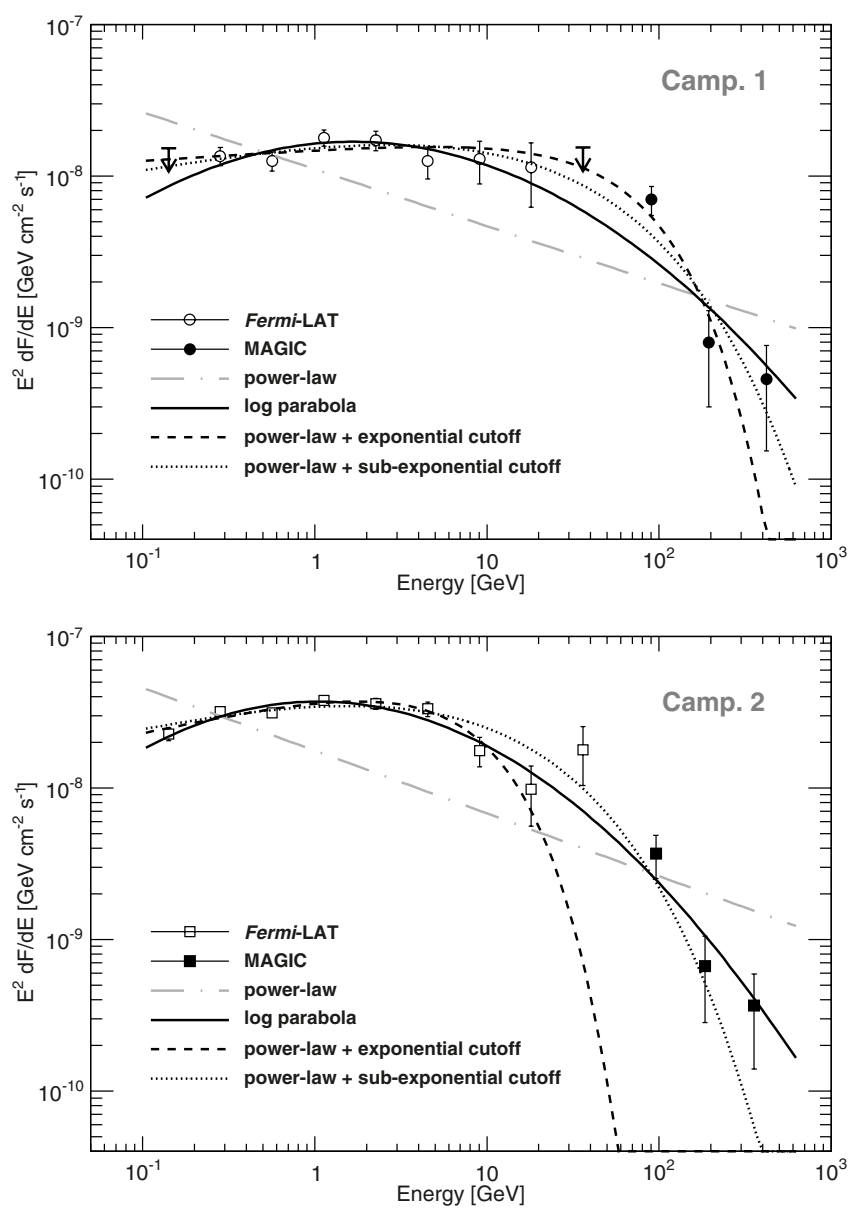

Fig. 9. NGC 1275 SED in $\gamma$-ray band measured with MAGIC and Fermi-LAT together with fit functions of the data (see parameters in Table 3). Upper and lower panels correspond to the periods October 2009-February 2010 and August 2010-February 2011, respectively.

We fitted several functions to these SEDs, taking into account only the statistical errors of each data point. Table 3 gives the functional forms used and the fit results.

A power-law fit is completely excluded, as suggested by previous VHE upper limits contemporaneous to Fermi-LAT data in 2008 (Acciari et al. 2009; Aleksić et al. 2010b). A powerlaw spectrum with an exponential cutoff fits the first campaign well but does not match the second one, where the LAT data clearly show a curvature. Assuming a softer cutoff (a powerlaw with a subexponential cutoff), one can fit both campaigns quite well. This gives a photon index of $\Gamma \simeq 1.75$ at low energy, which matches well the nonthermal power-law component seen in X-ray by Chandra (Balmaverde et al. 2006) and Swift-BAT (Ajello et al. 2009). As explained in Acciari et al. (2009), this function corresponds to the $\gamma$-ray spectral shape expected for the inverse Compton emission of an electron population with an energy distribution following a power law with an exponential cutoff at high energy ${ }^{6}$.

Finally, a curved power-law (log parabola) provides the best fit for Camp. 2 data and a reasonably good fit for Camp. 1. It

6 Strictly speaking, a subexponential description for the cutoff of the inverse Compton spectrum is valid only in the Thomson regime. As discussed in Sect. 3.4, the scattering responsible for emission at the highenergy peak in NGC 1275 mainly occurs in the Klein-Nishina regime. In this case, that particular shape can still be used as an approximation. 


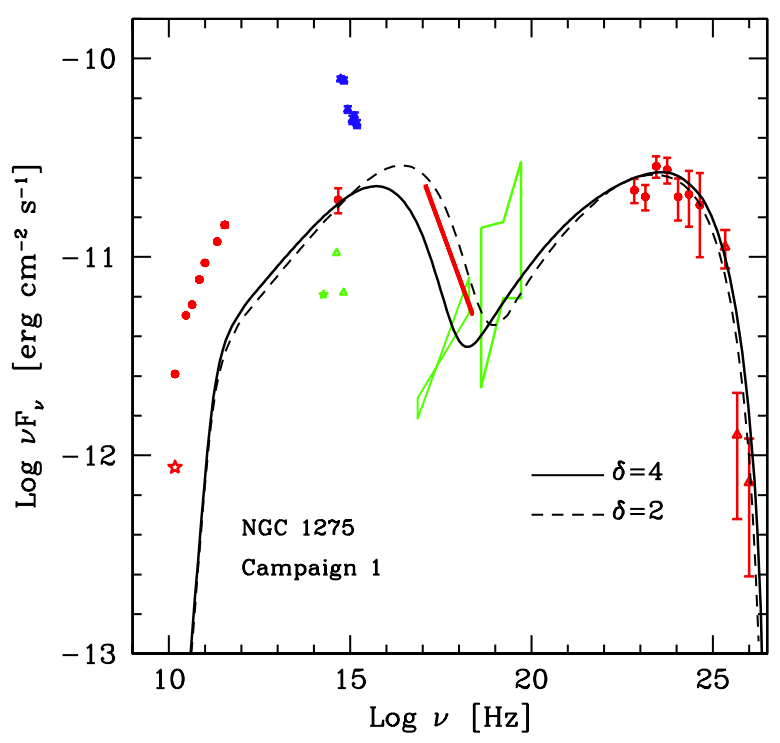

Fig. 10. NGC 1275 SED for the epoch of Camp. 1 (October 2009-February 2010). Red symbols show nearly simultaneous data from MOJAVE, KVA, Chandra (when assuming a power-law slope $\Gamma=2.5$ ), Fermi-LAT, and MAGIC. Contemporaneous Planck data from the Planck Collaboration (2011) are also shown with red circles. The red star in radio shows the level of the individual subparsec components $(\mathrm{C} 1, \mathrm{C} 2$, and $\mathrm{C} 3$ have nearly the same flux). Green bow-ties in the X-ray band report the archival Chandra and Swift-BAT results from Balmaverde et al. (2006) and Ajello et al. (2009), respectively. Green symbols report optical-IR measurement by HST from Chiaberge et al. (1999) (triangles) and Baldi et al. (2010) (star). The blue points show the August 2010 Swift-UVOT data from Giommi et al. (2012) (corrected for the host galaxy contribution). The dashed and solid lines report the SSC models for $\delta=2\left(\theta_{v}<30^{\circ}\right)$ and $\delta=4\left(\theta_{v}<15^{\circ}\right)$, respectively.

allows us to determine the peak energy of the SED, which varies marginally from $2.8 \pm 1.5 \mathrm{GeV}$ (Camp. 1) to $1.1 \pm 0.2 \mathrm{GeV}$ (Camp. 2).

\subsection{Broadband SED and emission models}

Nearly simultaneous SEDs for Camp. 1 and Camp. 2, assembled with the data described in the previous section, are reported in Fig. 10 (red symbols) and Fig. 11 (blue symbols), respectively. The nonthermal X-ray component spectra from Balmaverde et al. (2006) (Chandra) and Ajello et al. (2009) (Swift-BAT), as well as the optical-IR measurement by HST (Chiaberge et al. 1999; Baldi et al. 2010), are also reported (green symbols).

The data hint at a double-peaked SED, similar to the other TeV-emitting AGNs. The similarity of this SED with that of blazars is a clear indication that the broadband emission arises in the relativistic jet. The available data provide an excellent description of the high-energy bump of the SED. In particular, it is possible to constrain the position of the peak with good accuracy. On the other hand, the low-energy bump is poorly constrained by our radio-to-UV simultaneous data, which are more likely dominated by larger scale emission regions. The correlation between the optical and the $\gamma$-ray LC suggests that the nonthermal continuum from the NGC 1275 nucleus is dominated by the emission from the same region. We assume that the low-energy component of this region is at the level of our KVA measurements corrected for the host-galaxy and line emissions.

For our model, we assumed, as a starting point, that the emission is coming from a unique uniform spherical region (a bulk plasma) moving along the jet. We reproduced the SED

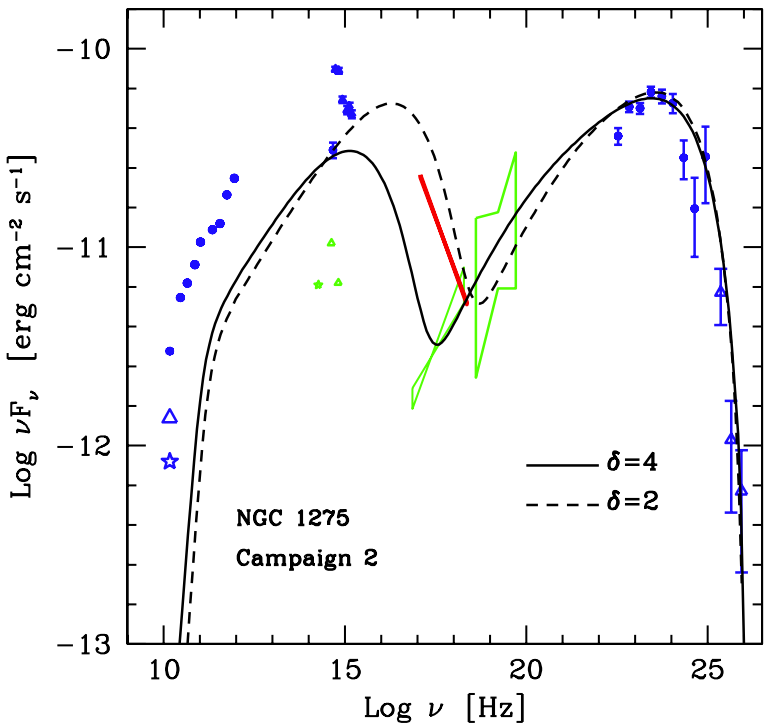

Fig. 11. NGC 1275 SED for the epoch of Camp. 2 (August 2010-February 2011). Symbols as in Fig. 10. Blue symbols show nearly simultaneous data from MOJAVE, KVA, Fermi-LAT and MAGIC analyzed here, and contemporaneous Swift-UVOT and Planck data from Giommi et al. (2012). In radio, the level of the subparsec components $\mathrm{C} 1$ (blue star) and C3 (blue triangle) are also shown.

using a synchrotron self-Compton (SSC) model (for details see Maraschi \& Tavecchio 2003) considering an electron-density energy distribution of the form $N(\gamma)=K \gamma^{-n} \exp \left(-\gamma / \gamma_{\mathrm{c}}\right)$, with a Lorentz factor $\gamma>\gamma_{\min }$. Such a very simple distribution is naturally expected in the case of Fermi-I type shock acceleration (e.g. Blandford \& Eichler 1987) and it is also supported by the $\gamma$-ray SED fits of Sect. 3.3. The other physical parameters specifying the model are the intensity of the magnetic field, $B$ (assumed to be tangled), the source radius, $R$, and the Doppler factor, $\delta$. The latter parameter is given by $\delta=1 /\left[\Gamma_{\mathrm{b}}\left(1-\beta_{\mathrm{b}} \cos \theta_{v}\right)\right]$, where $\beta_{\mathrm{b}}$ is bulk speed of the plasma, $\Gamma_{\mathrm{b}}$ is the bulk Lorentz factor, $\Gamma_{\mathrm{b}}=\left[1-\beta_{\mathrm{b}}^{2}\right]^{-1 / 2}$, and $\theta_{v}$ is the angle between the bulk velocity and the line of sight. Our model accordingly had only seven independent parameters $\left(K, n, \gamma_{\min }, \gamma_{\mathrm{c}}, B, R\right.$, and $\left.\delta\right)$.

Following the radio counter-jet evidence (Vermeulen et al. 1994; Walker et al. 1994; Asada et al. 2006), we first assumed an observing angle $\theta_{v}=30^{\circ}$, which is the lowest angle compatible with the radio observations. The highest value of the Doppler factor allowed was then $\delta=2$, reached for a bulk Lorentz factor $\Gamma_{\mathrm{b}}=2$. After fixing the Doppler factor, the source size can be constrained by the observed variability timescale in the FermiLAT band, $t_{\mathrm{var}} \simeq 1$ week, which limits the radius through the causality relation $R<c t_{\mathrm{var}} \delta \simeq 4 \times 10^{16} \mathrm{~cm}$, rather similar to the sizes used in most blazar models. During Camp. 1 the FermiLAT LC is smoother and the constraint on the radius must be relaxed to light-month scales: $R / \delta<10^{17} \mathrm{~cm}$.

Some estimates of the other physical parameters can be derived with simple arguments. While the peak of the SSC bump arises from scattering in the Klein-Nishina (KN) regime, its frequency can be directly used to estimate the Lorentz factor $\gamma_{\mathrm{c}}$ of the electrons at the high-energy cutoff. Following Tavecchio et al. $(1998)^{7}$, the frequency of the SSC peak, $v_{\mathrm{SSC}}$, is given as

7 Tavecchio et al. (1998) considered an electron energy distribution with a broken power-law shape. The results can be extended to the present case of a power law with exponential cutoff by identifying the break parameter $\gamma_{\mathrm{b}}$ with the cutoff parameter $\gamma_{\mathrm{c}}$ and taking the limit $\alpha_{1}=\alpha$ and $\alpha_{2} \gg 1$. 
Table 4. Parameters for the models reported in Figs. 10, 11.

\begin{tabular}{cccccccc}
\hline \hline & $\delta$ & $\begin{array}{c}B \\
{[\mathrm{mG}]}\end{array}$ & $\begin{array}{c}K \\
{\left[10^{4} \mathrm{~cm}^{-3}\right]}\end{array}$ & $\begin{array}{c}R \\
{\left[10^{16} \mathrm{~cm}\right]}\end{array}$ & $\gamma_{\min }$ & $\gamma_{\mathrm{c}}$ & $n$ \\
\hline Camp. 1 & 4 & 42 & 20 & 7.9 & 100 & $2.2 \times 10^{5}$ & 2.55 \\
& 2 & 170 & 18 & 8.2 & 100 & $3.5 \times 10^{5}$ & 2.55 \\
\hline Camp. 2 & 4 & 38 & 9 & 8.2 & 100 & $0.9 \times 10^{5}$ & 2.4 \\
& 2 & 380 & 14.7 & 4.1 & 100 & $1.5 \times 10^{5}$ & 2.4 \\
\hline
\end{tabular}

Notes. For both epochs the table reports the result obtained for two different choices of Doppler factor, $\delta=2$ and 4 . The parameters are the magnetic field $B$, the particle density $K$, the source radius $R$, the minimum electron Lorentz factor, $\gamma_{\min }$, the cutoff Lorentz factor $\gamma_{\mathrm{c}}$, and the electron spectrum slope $n$.

$h v_{\mathrm{SSC}} \simeq m_{\mathrm{e}} c^{2} \gamma_{\mathrm{c}} g(\alpha) \delta$, where $g(\alpha)=\exp [1 /(\alpha-1)]$ is a function of the energy-flux spectral slope of the rising edge of the synchrotron and inverse Compton bumps, $\alpha=(n-1) / 2$. The fit of the SSC bump with a power-law with a subexponential cutoff, reported in Sect. 3.3, provides a good estimation of the rising edge slope $\alpha \simeq 0.75 \pm 0.1$ (the fit parameter $\Gamma$ is the power-law index of the photon flux, $\Gamma=\alpha+1$ ). This value also agrees well with the spectral slope measured in hard X-rays with SwiftBAT ( $\alpha=0.7_{-0.7}^{+0.3}$, Ajello et al. 2009). For $\alpha=0.75$, the value of $g \simeq 0.02$. The SSC peak is measured around $v_{\mathrm{SSC}} \simeq 10^{24} \mathrm{~Hz}, \mathrm{im}-$ plying $\gamma_{\mathrm{c}} \simeq 2 \times 10^{5}$. The frequency of the synchrotron peak $v_{\mathrm{s}}$ is now found as $v_{\mathrm{s}} \simeq 2.8 \times 10^{6} \mathrm{~B} \gamma_{\mathrm{c}}^{2} \delta \mathrm{Hz}$. The condition (Tavecchio et al. 1998) that the SSC peak is produced by scatterings in the $\mathrm{KN}$ regime is $h \gamma_{\mathrm{c}} \nu_{\mathrm{s}} / \delta>m_{\mathrm{e}} c^{2}$, which, after some operations, can be expressed as a limit for the magnetic field, $B>B_{\mathrm{cr}} \gamma_{\mathrm{c}}^{-3} \mathrm{G}$, where $B_{\mathrm{cr}}=4.4 \times 10^{13} \mathrm{G}$ is the critical magnetic field. Inserting the value above for $\gamma_{\mathrm{c}}$, we found $B>4 \times 10^{-3} \mathrm{G}$.

An estimate of the magnetic field can be derived from the ratio between the synchrotron and $\mathrm{SSC}$ luminosities, $L_{\mathrm{S}} / L_{\mathrm{SSC}} \simeq$ $U_{B} / U_{\text {rad }}$, where $U_{B}=B^{2} / 8 \pi$ is the magnetic energy density and $U_{\text {rad }}$ is the synchrotron radiation energy density (Tavecchio et al. 1998). The latter can be calculated from $U_{\text {rad }} \simeq L_{\mathrm{s}} /\left(4 \pi R^{2} c \delta^{4}\right)$. Assuming the SSC emission occurs in the $\mathrm{KN}$ regime reduces the radiation energy density available for the emission. However, in first approximation one can neglect this effect (this derived value is formally a lower limit of the magnetic field). Finally, the magnetic field can be expressed as $B \simeq(2 / c)^{1 / 2} R^{-1} \delta^{-2} L_{\mathrm{S}} L_{\mathrm{SSC}}^{-1 / 2}$. Deriving the luminosities from the SED and using $R=4 \times$ $10^{16} \mathrm{~cm}$, we obtain $B \simeq 0.1 \mathrm{G}$. This value is well above the limit for the $\mathrm{KN}$ regime calculated above, confirming the previous assumption.

Table 4 reports the parameters obtained for the emission model, shown as dashed curves in Figs. 10, 11. The frequency of the synchrotron peak is relatively high $\left(>10^{16} \mathrm{~Hz}\right)$ and the model predicts soft X-ray emission dominated by the end of the synchrotron hump. The expected X-ray flux is much higher than the archival Chandra data and shows a very different spectral shape. This would rule out our model if the data were simultaneous. However, this Chandra observation (ObsID 3404) was taken in 2002 , long before the renewed activity in radio band started in 2005, and the X-ray emission may have changed dramatically. Compared with the contemporaneous Chandra data analyzed in Sect. 2.3, our SED model for Camp. 1 agrees well when one assumes (as must be done because the strong pileup does not allow us to estimate it) a photon index $\Gamma=2.5$ as expected from the model. For Camp. 2, the expected X-ray flux is even higher and the UV emission almost reaches the Swift-UVOT data. The simultaneous data do not rule out our model but the overall shape with a synchrotron peak in X-ray seems unlikely. The X-ray flux has never been measured at such a high level. A lower synchrotron peak frequency (in optical) would be more natural. It is very important to know the slope of the X-ray spectrum to determine the position of the synchrotron peak. Unfortunately, the quality of the contemporaneous Chandra data, which are strongly affected by pileup, does not allow us to derive strong constraints. An X-ray emission from either synchrotron $(\Gamma=\sim 2.5)$ or inverse Compton $(\Gamma=\sim 1.7)$ is possible.

As discussed in Tavecchio \& Ghisellini (2008) and Aleksić et al. (2010b), a small "distance" between the SED peaks is unavoidable for low Doppler factors. In order to increase the separation between the peaks, which would shift the synchrotron bump to lower frequencies, we are forced to increase $\delta$. In fact, using the relations developed above, it is relatively easy to see that (for a fixed $v_{\mathrm{SSC}}$ ) the synchrotron peak frequency is related to the corresponding Doppler factor by $v_{\mathrm{s}} \propto \delta^{-4}$. In Figs. 10-11 and Table 4, we also report the models for a Doppler factor that is twice as large, $\delta=4$ (solid lines). According to the scaling above, the synchrotron peak frequency decreases by more than a decade. The optical flux remains at the same level, while the UV and X-ray emission decrease significantly.Our model with $\delta=4$ would be almost compatible with the hard X-ray spectrum reported in Balmaverde et al. (2006). This value of the Doppler factor is disfavored, however, since it requires an angle smaller than $15^{\circ}$ between the plasma bulk velocity and the line of sight.

\section{Discussion}

The overall SED of NGC 1275 suggests blazar-like emission. A one-zone SSC model can fit the data assuming a jet-viewing angle $\left(\theta_{v}=30^{\circ}\right)$ compatible with the radio observation. All parameters (possibly with the exception of the bulk Lorentz factor $\Gamma_{b}$ ) derived from this modeling are in the range of the values typically obtained for VHE $\gamma$-ray emitting BL Lac objects (e.g., Tavecchio et al. 2010). Another interesting remark concerns the required slope of the electron energy distribution, which is close to 2.5. This value agrees well with recent simulations by Sironi \& Spitkovsky (2011), who showed that for low values of the jet magnetization (such as those derived by our SED modeling) Fermi-I shock acceleration proceeds efficiently, leading to power-law tails with slope $n=2.5$.

All these elements support the view that the $\gamma$-ray emission of NGC 1275 originates from a misaligned BL Lac jet, in the same way as for the other VHE $\gamma$-ray emitting radio galaxies, and consistent with the unification scheme (e.g., Urry \& Padovani 1995). A notable exception, however, is the bulk Lorentz factor, constrained to have a lower value $\left(\Gamma_{\mathrm{b}} \simeq 2\right)$ than 
the typical $\Gamma_{\mathrm{b}}>10$ inferred in BL Lacs. Indeed, with a jetviewing angle $\theta_{v} \simeq 30^{\circ}$, a larger $\Gamma_{\mathrm{b}}$ would imply a smaller Doppler factor, while our model with $\delta=2$ already seems at the lower edge of the acceptable range (a lower $\delta$ would imply a smaller distance between the two SED peaks, inducing a too high X-ray flux). Such a low $\Gamma_{\mathrm{b}}$ would indicate either that the jet of NGC 1275 is ejected at a lower speed than in blazars, or that the jet suffers strong acceleration/deceleration in the vicinity of the central engine. The kinematics of the radio knots might suggest acceleration along the NGC 1275 jet (Dhawan et al. 1998). Its $\gamma$-ray emitting region might then be closer to the central black hole than for typical blazars (before the bulk is accelerated to its full speed).

However, a larger bulk Lorentz factor would be possible with a smaller jet-sight angle $\theta_{v}$. For example, a bulk emitting region with $\Gamma_{\mathrm{b}}=10$ would be seen with a Doppler factor $\delta=2$ for $\theta_{v}=17^{\circ}$ and with $\delta=4$ for $\theta_{v}=12^{\circ}$. In the past, a smaller viewing angle has been suggested for the innermost jet (Krichbaum et al. 1992; Veron 1978). The NGC 1275 core could then be a BL Lac with strong jet bending at larger scale. The high energy nonthermal continuum of NGC 1275 could be also dominated by a substructure of the jet that points closer to our direction than the overall radio jet. Such scenarios have previously been proposed to explain the $\gamma$-ray emission from other $\mathrm{TeV}$ radio galaxies (M87 and Cen $\mathrm{A}$ ). This substructure could originate from the jet formation zone near the central black-hole where the jet is less collimated (Lenain et al. 2008), or from mini-jets in the main jet induced by magnetic reconnections (Giannios et al. 2010; Cui et al. 2012). Alternatively, the emission can arise in a magneto-centrifugally accelerated flow (e.g., Rieger \& Mannheim 2002), whose weak collimation would be compatible with the large viewing angles inferred for radio galaxies.

One may notice that our model predicts a much lower flux at $15 \mathrm{GHz}$ than the VLBA measurements, even in a single substructure. This is related to the relatively high energy peak of the synchrotron component. The radio emission could come from another, more external, region where the low-energy electrons could diffuse. The variability of such an external component should be much smoother. The similar long-term trend of the $\mathrm{C} 3$ component and the $\mathrm{GeV} \gamma$-ray emission makes it a suitable candidate for hosting the $\gamma$-ray emitting region. However, the apparent velocity of this component, $\beta_{\text {app }}=0.23$ (Nagai et al. $2010)$, is much too slow compared with the apparent velocity of the emitting region of our one-zone SSC model. For $\theta_{v}=30^{\circ}$ and $\Gamma_{\mathrm{b}}=2$, the expected apparent velocity is superluminal, $\beta_{\text {app }}=\frac{\beta_{\mathrm{b}} \sin \left(\theta_{v}\right)}{1-\beta_{\mathrm{b}} \cos \left(\theta_{v}\right)} \simeq 1.7$. The models with smaller viewing angle would have even larger $\beta_{\text {app }}$. Indeed, the $\mathrm{C} 3$ radio component and the $\gamma$-ray source may not be co-spatial but the increasing emission in both may simply be connected to a general increasing activity of the AGN. Moreover, no clear correlation on short timescales was found by Nagai et al. (2012).

The difficulty in determining the correct bulk Lorentz factor of the jet is not specific to NGC 1275 but is a general problem of the AGN unification scheme, the so-called bulk Lorentz factor crisis (Henri et al. 2006). While one-zone SSC models of $\mathrm{TeV}$ blazars require a large Lorentz factor $\left(\Gamma_{\mathrm{b}}>10\right)$, geometrical beaming arguments on FR I radio galaxy - BL Lac unification predict a low value $\left(\Gamma_{\mathrm{b}} \simeq 3\right.$ ) (Chiaberge et al. 2000). More complex models that assume, for example, a structured jet with different emission zones (Ghisellini et al. 2005) or blob-speed changes along the jet (Georganopoulos \& Kazanas 2003), must be developed to solve this crisis. The simultaneous broadband observations of NGC 1275 reported here provide a rare view of a very likely misaligned BL Lac object. They provide new constraints on models developed to reconcile the VHE $\gamma$-ray observations and the unification scheme of AGNs.

\section{Summary and conclusion}

We analyzed multifrequency observations of NGC 1275 contemporaneous to two MAGIC observation campaigns carried out from October 2009 to February 2011. MAGIC data analysis, with an energy threshold of $100 \mathrm{GeV}$, resulted in the detection of the source during both campaigns separately, confirming that NGC 1275 belongs to the very exclusive club of radio galaxies detected at VHE. The $\sim 65-650 \mathrm{GeV}$ spectra measured with MAGIC are very similar for both campaigns. The monthly VHE LC shows only a hint of variability $(3.6 \sigma)$ during the first campaign. Fermi-LAT results between $100 \mathrm{MeV}$ and $\sim 50 \mathrm{GeV}$ present different features. The LAT LC shows a clear week-scale variability, and the two derived spectra, corresponding to each of the MAGIC campaigns, show differences in both flux intensity and spectral shape. Nevertheless, the combined LAT/MAGIC spectra can be well fit by both a log parabola and a power law with a subexponential cutoff. The SED-peak energies of the log parabolic fits are $2.8 \pm 1.5 \mathrm{GeV}$ (Camp. 1) and $1.1 \pm 0.2 \mathrm{GeV}$ (Camp. 2). The subexponential cutoff energies are $13.1 \pm 4.2 \mathrm{GeV}$ (Camp. 1) and 5.0 $\pm 1.6 \mathrm{GeV}$ (Camp. 2). The rapid variability and spectral evolution imply that the $\gamma$-ray emitting region is a compact zone in the AGN. The KVA $R$-band LC, corrected for host-galaxy and emission-line contaminations, shows a shape very similar to the Fermi-LAT LC. This almost linear correlation strongly suggests that the optical counterpart of the very compact $\gamma$-ray emitting region dominates the corrected KVA result. The $15 \mathrm{GHz}$ MOJAVE data allowed us to derive the LC of the three innermost radio components. The recently born $\mathrm{C} 3$ component shows a similar LC trend as the KVA and LAT LCs. This makes this jet feature a possible candidate for the $\gamma$-ray emitting region.

We modeled the simultaneous broadband SED with a simple one-zone SSC emission model assuming a power-law with an exponential cutoff for the electron energy distribution. While the high-energy peak is well constrained by the LAT and MAGIC data, at low energy most of our simultaneous data must be considered as upper limits due to possible contaminations. We assumed as optical flux the corrected KVA data, which show correlation with the $\gamma$-ray flux. The relatively large angle between the NGC 1275 jet and the line of sight $\left(\theta_{v}=30^{\circ}-55^{\circ}\right)$ strongly limits the Doppler factor $\delta$. A model with $\delta=2\left(\theta_{v}=30^{\circ}\right.$ and $\left.\Gamma_{\mathrm{b}}=2\right)$ can fit the SED of both epochs and explain the multifrequency behavior (optical-GeV correlation and rapid variability). However, the expected X-ray level is in contrast with the Chandra measurements and models with lower $\delta$ would hardly match the data as they would imply a too small spread between the low- and high-energy SED peaks. On the other hand, models with larger $\delta$ (such as $\delta=4$ ) would fit the SED well, but would require smaller $\theta_{v}$. In fact, the parameters of our models are in the typical range found for BL Lacs, except for the bulk Lorentz factor. NGC 1275 might accordingly be a misaligned BL Lac with a particularly low bulk Lorentz factor, or it might be more aligned than we think. Assuming a smaller $\theta_{v}\left(10-15^{\circ}\right)$, larger bulk Lorentz factors $\left(\Gamma_{\mathrm{b}}>10\right)$ are possible. A $\gamma$-ray emitting region with a velocity more aligned to the line of sight than the parsec-scale radio jet could be explained by jet bending in the innermost region, a larger jet opening angle near the central black hole, or mini-jets within the main jet. Multizone 
models would certainly be more appropriate for interpreting the NGC 1275 AGN emission and its connection to BL Lac objects.

Acknowledgements. The MAGIC collaboration would like to thank the Instituto de Astrofísica de Canarias for the excellent working conditions at the Observatorio del Roque de los Muchachos in La Palma. The support of the German BMBF and MPG, the Italian INFN, the Swiss National Fund SNF, and the Spanish MICINN is gratefully acknowledged. This work was also supported by the CPAN CSD2007-00042 and MultiDark CSD2009-00064 projects of the Spanish Consolider-Ingenio 2010 programme, by grant DO02-353 of the Bulgarian NSF, by grant 127740 of the Academy of Finland, by the DFG Cluster of Excellence "Origin and Structure of the Universe", by the DFG Collaborative Research Centers SFB823/C4 and SFB876/C3, and by the Polish MNiSzW grant 745/N-HESS-MAGIC/2010/0. The Fermi-LAT Collaboration acknowledges ongoing support from a number of agencies and institutes that have supported both the development and the operation of the LAT as well as scientific data analysis. These include the National Aeronautics and Space Administration and the Department of Energy in the United States, the Commissariat à l'Énergie Atomique and the Centre National de la Recherche Scientifique/Institut National de Physique Nucléaire et de Physique des Particules in France, the Agenzia Spaziale Italiana and the Istituto Nazionale di Fisica Nucleare in Italy, the Ministry of Education, Culture, Sports, Science and Technology (MEXT), High Energy Accelerator Research Organization (KEK) and Japan Aerospace Exploration Agency (JAXA) in Japan, and the K. A. Wallenberg Foundation, the Swedish Research Council, and the Swedish National Space Board in Sweden. Additional support for science analysis during the operations phase is gratefully acknowledged from the Istituto Nazionale di Astrofisica in Italy and the Centre National d'Études Spatiales in France. This research has made use of data from the MOJAVE database that is maintained by the MOJAVE team (Lister et al. 2009).

\section{References}

Abdo, A. A., Ackermann, M., Ajello, M., et al. (Fermi collaboration) 2009, ApJ, 699, 31

Acciari, V. A., Aliu, E., Arlen, T., et al. (VERITAS collaboration) 2009, ApJ, 706, L275

Ackermann, M., Ajello, M., Albert, A., et al. (Fermi collaboration) 2012, ApJS, 203, 4

Ajello, M., Rebusco, P., Cappelluti, N., et al. 2009, ApJ, 690, 367

Aleksić, J., Antonelli, L. A., Antoranz, P., et al. (MAGIC collaboration) 2010a, ApJ, 723, L207

Aleksić, J., Antonelli, L. A., Antoranz, P., et al. (MAGIC collaboration) 2010b, ApJ, 710, 634

Aleksić, J., Alvarez, E. A., Antonelli, L. A., et al. (MAGIC collaboration) 2012a, A\&A, 539, L2

Aleksić, J., Alvarez, E. A., Antonelli, L. A., et al. (MAGIC collaboration) 2012b, A\&A, 541, A99

Aleksić, J., Alvarez, E. A., Antonelli, L. A., et al. (MAGIC collaboration) 2012c, Astropart. Phys., 35, 435

Aleksić, J., Antonelli, L. A., Antoranz, P., et al. (MAGIC collaboration) 2014, A\&A, 563, A91

Aliu, E., Anderhub, H., Antonelli, L. A., et al. (MAGIC collaboration) 2009, Astropart. Phys., 30, 293

Asada, K., Kameno, S., Shen, Z.-Q., et al. 2006, Publ. Astron. Soc. Japan, 58, 261

Atwood, W. B., Abdo, A. A., Ackermann, M., et al. 2009, ApJ, 697, 1071

Baldi, R. D., Chiaberge, M., Capetti, A., et al. 2010, ApJ, 725, 2426

Balmaverde, B., Capetti, A., Grandi, P., et al. 2006, A\&A, 451, 35

Blandford, R., \& Eichler, D. 1987, Phys. Rep., 154, 1

Brown, A. M., \& Adams, J. 2011, MNRAS, 413, 2785

Caulet, A., Woodgate, B. E., Brown, L. W., et al. 1992, ApJ, 388, 301

Celotti, A., \& Ghisellini, G. 2008, MNRAS, 385, 283

Chiaberge, M., Capetti, A., \& Celotti, A. 1999, A\&A, 349, 77

Chiaberge, M., Celotti, A., Capetti, A., \& Ghisellini, G. 2000, A\&A, 358, 104

Churazov, E., Forman, W., Jones, C., \& Böhringer, H. 2003, ApJ, 590, 225

Conselice, C. J., Gallagher, J. S., \& Wyse, R. F. G. 2001, AJ, 122, 2281

Colafrancesco, S., Marchegiani, III, P., \& Giommi, P. 2010, A\&A, 519, A82

Cui, Y.-D., Yuan, Y.-F., Li, Y.-R., \& Wang, J.-M. 2012, ApJ, 746, 177

Davis, J. E. 2001, ApJ, 562, 575

Dhawan, V., Kellermann, K. I., \& Romney, J. D. 1998, ApJ, 498, L111

Eckert, D., \& Paltini, S. 2009, A\&A, 495, 415

Edelson, R. A., \& Krolik, J. H. 1988 ApJ, 333, 646

Fabian, A. C., Sanders, J. S., Allen, S. W., et al. 2011, MNRAS, 418, 2154

Fiorucci, M., Tosti, G., \& Rizzi, N. 1998, PASP, 110, 105
Fomin, V. P., Stepanian, A. A., Lambet, R. C., et al. 1994, Astropart. Phys., 2, 137

Ghisellini, G., Tavecchio, F., \& Chiaberge, M. 2005, A\&A, 432, 401

Ghisellini, G., Tavecchio, F., Foschini, L., et al. 2010, MNRAS, 402, 497

Georganopoulos, M., \& Kazanas, D. 2003, ApJ, 594, L27

Giannios, D., Uzdensky, D. A., \& Begelman, M. C. 2010, MNRAS, 402, 1649

Giommi, P., Polenta, G., Lähteenmäki, A., et al. 2012, A\&A, 541, A160

Goetting, N., et al. (HEGRA Collaboration) 2001, in Proc. of 27th ICRC, Hamburg, 2669

Henri, G., \& Saugé, L. 2006 ApJ, 640, 185

Hillas, A. M. 1985, in Proc. of 19th ICRC, La Jolla, 445

Kataoka, J., Stawarz, L., Cheung, C. C., et al., 2010, ApJ, 715, 554

Krichbaum, T. P., Witzel, A., Graham, D. A., et al. 1992, A\&A, 260, 33

Lenain, J.-P., Boisson, C., Sol, H., \& Katarzynski, K. 2008, A\&A, 478, 111

Li, T.-P., \& Ma, Y.-Q. 1983, ApJ, 272, 317

Lister, M. L., Aller, H. D., Aller, M. F., et al. 2009, AJ, 137, 3718

Lombardi, S., Berger, K., Collin, P., Diago Ortega, A., \& Klepser, S. (MAGIC collaboration) 2011, Proc. of 32nd ICRC, Beijin, China, August [arXiv: 1109.6195]

Lynds, R. 1970, ApJ, 159, L151

Maraschi, L., \& Tavecchio, F. 2003, ApJ, 593, 667

Massaro, F., Tremblay, G. R., Harris, D. E., et al. 2012, ApJS, 203, 31

Nagai, H., Suzuki, K., Asada, K., et al. 2010, Publ. Astron. Soc. Japan, 62, L11

Nagai, H., Orienti, M., Kino, M., et al. 2012, MNRAS, 423, L122

Nagai, H., Kino, M., Niinuma, K., et al. 2013, Publ. Astron. Soc. Japan, 65, 24

Nilsson, K., Pasanen, M., Takalo, L. O., et al. 2007, A\&A, 475, 199

Nolan, P. L., Abdo, A. A., Ackermann, M., et al. 2012, ApJS, 199, 31

Planck Collaboration, et al. 2011, A\&A, 536, A7

Pedlar, A., Ghataure, H. S., Davies, R. D., et al. 1990, MNRAS, 246, 477

Perkins, J. S., Badran, H. M., Blaylock, G., et al. (VERITAS Collaboration) 2006, ApJ, 644, 148

Pinzke, A., \& Pfrommer, C. 2010, MNRAS, 409, 2, 449

Reimer, O., Pohl, M., Sreekumar, P., \& Mattox, J. R. 2003, ApJ, 588, 155

Rieger, F. M., \& Mannheim, K. 2002, A\&A, 396, 833

Rolke, W. A., López, A. M., \& Conrad, J. 2005, Nucl. Instrum. Meth. A, 551, 493

Schlegel, D. J., Finkbeiner, D. P., \& Davis, M. 1998, ApJ, 500, 525

Seyfert, C. K. 1943, ApJ, 97, 28

Sironi, L., \& Spitkovsky, A. 2011, ApJ, 726, 75

Strong A. W., \& Bignami G. F. 1983 ApJ, 274, 549

Tavecchio, F., \& Ghisellini, G. 2008, MNRAS, 385, L98

Tavecchio, F., Maraschi, L., \& Ghisellini, G. 1998, ApJ, 509, 608

Tavecchio F., Ghisellini G., Ghirlanda G., Foschini L., \& Maraschi L., 2010, MNRAS, 401, 1570

Urry, C. M., \& Padovani, P. 1995, PASP, 107, 803

Vermeulen, R. C., Readhead, A. C. S., \& Backer, D. C. 1994, ApJ, 430, L41

Veron, P. 1978, Nature, 272, 430

Walker, R. C., Romney, J. D., \& Benson, J. M. 1994, ApJ, 430, L45

1 IFAE, Edifici Cn., Campus UAB, 08193 Bellaterra, Spain

2 Università di Udine, and INFN Trieste, 33100 Udine, Italy

3 INAF National Institute for Astrophysics, 00136 Rome, Italy

4 Università di Siena, and INFN Pisa, 53100 Siena, Italy

5 Croatian MAGIC Consortium, Rudjer Boskovic Institute, University of Rijeka and University of Split, 10000 Zagreb, Croatia

6 Max-Planck-Institut für Physik, 80805 München, Germany

7 Universidad Complutense, 28040 Madrid, Spain

8 Inst. de Astrofísica de Canarias, 38200 La Laguna, Tenerife, Spain

9 University of Lodz, 90236 Lodz, Poland

10 Deutsches Elektronen-Synchrotron (DESY), 15738 Zeuthen, Germany

11 ETH Zurich, 8093 Zurich, Switzerland

12 Universität Würzburg, 97074 Würzburg, Germany

13 Centro de Investigaciones Energéticas, Medioambientales y Tecnológicas, 28040 Madrid, Spain

14 Technische Universität Dortmund, 44221 Dortmund, Germany

15 Inst. de Astrofísica de Andalucía (CSIC), 18080 Granada, Spain

16 Università di Padova and INFN, 35131 Padova, Italy

17 Università dell'Insubria, Como, 22100 Como, Italy

18 Unitat de Física de les Radiacions, Departament de Física, and CERES-IEEC, Universitat Autònoma de Barcelona, 08193 Bellaterra, Spain

19 Institut de Ciències de 1'Espai (IEEC-CSIC), 08193 Bellaterra, Spain 
20 Finnish MAGIC Consortium, Tuorla Observatory, University of Turku and Department of Physics, University of Oulu, 900147 Oulu Finland

21 Japanese MAGIC Consortium, Division of Physics and Astronomy, Kyoto University, Japan

22 Inst. for Nucl. Research and Nucl. Energy, 1784 Sofia, Bulgaria

${ }^{23}$ Universitat de Barcelona (ICC/IEEC), 08028 Barcelona, Spain

24 Università di Pisa, and INFN Pisa, 56126 Pisa, Italy

25 Now at École polytechnique fédérale de Lausanne (EPFL), Lausanne, Switzerland

26 Now at Department of Physics \& Astronomy, UC Riverside CA 92521, USA
27 Now at Finnish Centre for Astronomy with ESO (FINCA), 21500 Turku, Finland

28 also at Instituto de Fisica Teorica, UAM/CSIC, 28049 Madrid, Spain

29 Now at Stockholms universitet, Oskar Klein Centre for Cosmoparticle Physics, Stockholm University, 10691 Stockholm Sweden

30 Now at GRAPPA Institute, University of Amsterdam, 1098XH Amsterdam, The Netherlands

31 INAF - Osservatorio Astrofisico di Torino, Strada Osservatorio 20, 10025 Pino Torinese, Italy

32 Waseda University, 169-8050, Tokyo, Japan 